\title{
Biorders with frontier
}

\author{
Denis Bouyssou ${ }^{1}$ \\ CNRS - LAMSADE \& \\ Université Paris Dauphine
}

Thierry Marchant ${ }^{2}$

Universiteit Gent

22 January 2010

Authors are listed alphabetically.

${ }^{1}$ CNRS, LAMSADE, FRE3234 \& Université Paris Dauphine, Place du Maréchal de Lattre de Tassigny, F-75775 Paris Cedex 16, France, tel: +33 1440548 98, fax: +331 440540 91, e-mail: bouyssou@lamsade.dauphine.fr, Corresponding author.

2 Universiteit Gent, Department of Data Analysis, H. Dunantlaan, 1, B-9000 Gent, Belgium, tel: +32 926463 73, fax: +32926464 87, e-mail: thierry.marchant@UGent. be. 


\begin{abstract}
This paper studies an extension of biorders that has a "frontier" between the relation and the absence of relation. This extension is motivated by a conjoint measurement problem consisting in the additive representation of ordered coverings defined on product sets of two components. We also investigate interval orders and semiorders with frontier.
\end{abstract}

Keywords: biorder, interval order, semiorder, frontier.

MSC (2010): 06A75 


\section{Introduction}

Let $\mathcal{T}$ be a relation between two sets $A$ and $Z$, i.e., a subset of $A \times Z$. Biorders are relations between two sets that were introduced in the literature by Ducamp and Falmagne (1969) and later studied in Doignon, Ducamp, and Falmagne (1984, 1987) and Nakamura (2002). The initial motivation for studying biorders was the formalization of the idea of a Guttman scale, i.e., a model in which there are real-valued functions $f$ on $A$ and $g$ on $Z$ such that, for all $a \in A$ and all $p \in Z$,

$$
a \mathcal{T} p \Leftrightarrow f(a)>g(p) .
$$

The usual interpretation is that $A$ is a set of subjects and $Z$ a set of questions. The relation $\mathcal{T}$ is defined in such a way that $a \mathcal{T} p$ if subject $a$ gives the correct answer to question $p$. If the above model obtains, the scale $f$ therefore orders subjects according to their ability to answer questions and the scale $g$ orders questions according to their difficulty. Biorders also proved an important tool to study various classes of binary relations, most notably interval orders and semiorders. Indeed, when $A=Z$, an irreflexive biorder is nothing but an interval order as defined in Fishburn (1970). Adding semitransitivity to irreflexivity leads to semiorders (Luce, 1956, Scott and Suppes, 1958).

This paper studies extensions of biorders in which we consider two relations $\mathcal{T}$ and $\mathcal{F}$ between the sets $A$ and $Z$. Using the interpretation of biorders introduced above, the relation $\mathcal{F}$ corresponds to situations in which we are unsure whether the subject has given or not the correct answer to the question. It therefore plays the role of a frontier between correct and incorrect answers. The underlying numerical model will be such that:

$$
\begin{aligned}
& a \mathcal{T} p \Leftrightarrow f(a)>g(p), \\
& a \mathcal{F} p \Leftrightarrow f(a)=g(p) .
\end{aligned}
$$

The main purpose of this paper is to study such structures, called biorders with frontier. Our initial motivation for doing so was not linked with Guttman scales but with a conjoint measurement problem encountered in the study of additive representations of ordered coverings on a product set (see Bouyssou and Marchant, $2009,2010)$. In this context, the frontier corresponds to a case of hesitation between two consecutive categories.

The paper is organized as follows. Section 2 presents our setting. Classic results on biorders are recalled in Section 3. Biorders with frontier are studied in Section 4. These results are used to tackle interval orders with frontier in Section 5. Section 6 is devoted to semiorders with frontier. Section 7 briefly presents our initial conjoint measurement motivation for studying structures with frontier and gives some applications of our results. 


\section{Definitions and Notation}

\subsection{Binary relations between two sets}

Let $A=\{a, b, \ldots\}$ and $Z=\{p, q, \ldots\}$ be two sets. Following Ducamp and Falmagne (1969), we define a binary relation $\mathcal{V}$ between $A$ and $Z$ to be a subset of $A \times Z$. We often write $a \mathcal{V} p$ instead of $(a, p) \in \mathcal{V}$. A binary relation on a set $X$ is a binary relation between $X$ and $X$.

Let $\mathcal{V}$ be a relation between $A$ and $Z$. Define ${ }^{1}$ the left trace of $\mathcal{V}$ as the binary relation $\succsim_{\mathcal{V}}^{\ell}$ on $A$ defined letting, for all $a, b \in A$,

$$
a \succsim^{\ell} b \Leftrightarrow[b \mathcal{V} p \Rightarrow a \vee p, \text { for all } p \in Z]
$$

Similarly, define the right trace of $\mathcal{V}$ as the binary relation $\succsim_{v}^{r}$ on $Z$ defined letting, for all $p, q \in Z$,

$$
p \succsim_{\mathcal{V}}^{r} q \Leftrightarrow[a \mathcal{V} p \Rightarrow a \mathcal{V} q, \text { for all } a \in A]
$$

By construction, the relations $\succsim_{\mathcal{V}}^{\ell}$ and $\succsim_{\mathcal{V}}^{r}$ are reflexive and transitive.

A binary relation $\mathcal{V}$ between $A$ and $Z$ is said to be a biorder if it is Ferrers, i.e., for all $a, b \in A$ and all $p, q \in Z$, we have:

$$
[a \mathcal{V} p \text { and } b \mathcal{V} q] \Rightarrow[a \mathcal{V} q \text { or } b \mathcal{V} p .]
$$

A simple check shows that $\mathcal{V}$ is Ferrers iff $\succsim_{v}^{\ell}$ is complete iff $\succsim_{v}^{r}$ is complete (see Doignon et al., 1984, Proposition 2, p. 78).

Let $\mathcal{V}$ be a relation between $A$ and $Z$. Its complement is the relation $\mathcal{V}^{c}$ between $A$ and $Z$ such that for all $a \in A$ and $p \in Z, a \mathcal{V}^{c} p \Leftrightarrow \operatorname{Not}[a \mathcal{V} p]$. The dual of $\mathcal{V}$ is the relation $\mathcal{V}^{d}$ between $Z$ and $A$ such that, for all $a \in A$ and $p \in Z$, $p \mathcal{V}^{d} a \Leftrightarrow a \mathcal{V}$. Its codual $\mathcal{V}^{c d}$ is the relation between $Z$ and $A$ such that, for all $a \in A$ and $p \in Z, p \mathcal{V}^{c d} a \Leftrightarrow \operatorname{Not}[a \mathcal{V} p]$. It is easy to check that if $\mathcal{V}$ is Ferrers iff $\mathcal{V}^{c d}$ (or $\mathcal{V}^{c}$, or $\mathcal{V}^{d}$ ) is Ferrers. The traces generated by $\mathcal{V}$ on $A$ and $Z$ coincide with the ones generated by $\mathcal{V}^{c d}$.

Suppose that $\mathcal{V}$ is a relation between $A$ and $Z$ and that $\mathcal{W}$ is a binary relation between $Z$ and $K$. We define the product of $\mathcal{V}$ and $\mathcal{W}$ as the binary relation $\mathcal{V W}$ between $A$ and $K$ such that, for all $a \in A$ and all $k \in K, a \mathcal{V W} k$ iff $^{2}[a \mathcal{V} p$ and $p \mathcal{W} k$, for some $p \in Z]$. The Ferrers property can therefore be expressed compactly as $\mathcal{V} \mathcal{V}^{c d} \mathcal{V} \subseteq \mathcal{V}$.

\footnotetext{
${ }^{1}$ Our use of the terms left trace and right trace differs from the one in Doignon, Monjardet, Roubens, and Vincke (1988). They use left trace to designate our right trace and vice versa. The rationale for our terminology is the following. A biorder is a subset of $A \times Z$. Hence, the set $A$ is the set "on the left". The left trace is a relation on the left set.

${ }^{2}$ i.e., if and only if
} 
For our purposes, when studying a relation between $A$ and $Z$, it is not restrictive to suppose that the sets $A$ and $Z$ are disjoint: if they are not, we may build a disjoint duplication of these sets as done in Doignon et al. (1984, Definition 4, p. 79). We will suppose so, without explicit mention, whenever needed.

\subsection{Binary relations on a set}

Let $V$ be a binary relation on $X$. The asymmetric part (resp. symmetric part, symmetric complement) of $V$ is the binary relation $V^{a}$ (resp. $V^{s}, V^{s c}$ ) on $X$ that is equal to $V \cap V^{c d}$ (resp. $V \cap V^{d}, V^{c} \cap V^{c d}$ ). For instance, we have $x V^{s c} y \Leftrightarrow$ $\left[x V^{c} y\right.$ and $\left.x V^{c d} y\right] \Leftrightarrow[\operatorname{Not}[x V y]$ and $\operatorname{Not}[y V x]]$.

Whenever we use the symbol $\succsim$ to denote a binary relation on a set $X$, it will be understood that $\succ$ (resp. $\sim$ ) denotes its asymmetric part (resp. symmetric part). The same convention will hold if $\succsim$ is subscripted and/or superscripted.

A binary relation that is complete (for all $x, y \in X, x V y$ or $y V x$ ) and transitive is said to be a weak order. If $V$ and $W$ are two weak orders on $X$, we say that $V$ refines $W$ if $V \subseteq W$. This implies $V^{s} \subseteq W^{s}$ and $W^{a} \subseteq V^{a}$.

The trace of a binary relation $V$ on $X$ is the binary relation $\succsim_{V}$ on $X$ that is equal to $\succsim_{V}^{\ell} \cap \succsim_{V}^{r}$. The relations $\succsim_{V}, \succsim_{V}^{\ell}$ and $\succsim_{V}^{r}$ are clearly reflexive, symmetric and transitive, i.e., are equivalences. Whenever $E$ is an equivalence on a set $X$, we denote by $X / E$ the set of equivalence classes of $X$ under $E$.

A binary relation $V$ on $X$ is said to be semitransitive if, for all $x, y, z, w \in X$,

$$
[x V y \text { and } y V z] \Rightarrow[x V w \text { or } w V z]
$$

which can be written more compactly as $V^{c d} V V \subseteq V$ or $V V V^{c d} \subseteq V$. A simple check shows that the trace $\succsim_{V}$ of a relation $V$ is complete iff $V$ is Ferrers and semitransitive. In this case the left and right traces are not contradictory, i.e., it is never true that $x \succ_{V}^{\ell} y$ and $y \succ_{V}^{r} x$, for some $x, y \in X$ (for more details, see, e.g., Fishburn, 1985, Monjardet, 1978, Pirlot and Vincke, 1997, or Suppes, Krantz, Luce, and Tversky, 1989, Ch. 16).

A binary relation $V$ on $X$ is an interval order if it is irreflexive and Ferrers. A semiorder is a semitransitive interval order. It is easy to check that an interval order is asymmetric, transitive, and such that $V V^{s c} V \subseteq V$. When, furthermore, $V$ is semitransitive, we have $V^{s c} V V \subseteq V$ and $V V V^{s c} \subseteq V$.

\section{Biorders, Interval Orders, Semiorders}

This section mainly recalls a number of basic results on the numerical representation of biorders, following Ducamp and Falmagne (1969), Doignon et al. (1984), 
and Nakamura (2002). We also briefly show how these results apply to interval orders and semiorders.

\subsection{Biorders: the countable case}

We first tackle the case in which both $A$ and $Z$ are countable (i.e., finite or countably infinite). We have:

Proposition 1 (Prop. 4, p. 79 in Doignon et al., 1984)

Let $A$ and $Z$ be finite or countably infinite sets and $\mathcal{T}$ be a relation between $A$ and

$Z$. The following statements are equivalent.

\section{1. $\mathcal{T}$ is Ferrers.}

2. There are a real-valued function $f$ on $A$ and a real-valued function $g$ on $Z$ such that, for all $a \in A$ and $p \in Z$,

$$
a \mathcal{T} p \Leftrightarrow f(a) \geq g(p) .
$$

3. There are a real-valued function $f$ on $A$ and a real-valued function $g$ on $Z$ such that, for all $a \in A$ and $p \in Z$,

$$
a \mathcal{T} p \Leftrightarrow f(a)>g(p)
$$

Furthermore, the functions $f$ and $g$ used in statements 2 or 3 above can always be chosen so that, for all $a, b \in A$ and $p, q \in Z$,

$$
\begin{aligned}
& a \succsim_{\mathcal{T}}^{\ell} b \Leftrightarrow f(a) \geq f(b), \\
& p \succsim_{\mathcal{T}}^{r} q \Leftrightarrow g(p) \geq g(q) .
\end{aligned}
$$

It may be instructive to outline the proof of the above result, following Doignon et al. (1984). Necessity is easily established. To prove sufficiency, let us build the binary relation $Q$ on $A \cup Z$ defined letting, for all $\alpha, \beta \in A \cup Z$,

$$
\alpha Q \beta \Leftrightarrow\left\{\begin{array}{l}
\alpha \in A, \beta \in A, \text { and } \alpha \succsim_{\mathcal{T}}^{\ell} \beta, \\
\alpha \in Z, \beta \in Z, \text { and } \alpha \succsim_{\mathcal{T}}^{r} \beta, \\
\alpha \in A, \beta \in Z, \text { and } \alpha \mathcal{T} \beta, \\
\alpha \in Z, \beta \in A, \text { and [for all } \gamma \in A, \delta \in Z, \gamma \mathcal{T} \alpha \text { and } \beta \mathcal{T} \delta \Rightarrow \gamma \mathcal{T} \delta \text { ]. }
\end{array}\right.
$$

It is easy to see that the relation $Q$ on $A \cup Z$ is always reflexive and transitive, i.e., is a quasi-order (Doignon et al., 1984, have shown that $Q$ is the maximal quasi-order on $A \cup Z$ that coincides with $\mathcal{T}$ on $A \times Z$ ). When $\mathcal{T}$ is a biorder, it is not difficult to check that relation $Q$ is also complete, so that it is a weak order. 
Since $A \cup Z$ is countable, there is a real-valued function $F$ on $A \cup Z$ representing $Q$, i.e., such that, for all $\alpha, \beta \in A \cup Z$,

$$
\alpha Q \beta \Leftrightarrow F(\alpha) \geq F(\beta) .
$$

Defining $f$ (resp. $g$ ) as the restriction of $F$ on $A$ (resp. $Z$ ) leads to a non-strict numerical representation of type (1). To obtain a strict numerical representation of type (2), it suffices to apply the same analysis to $\mathcal{T}^{c d}$.

As will be apparent later, $Q$ is not the only relation on $A \cup Z$ that is a weak order when $\mathcal{T}$ is Ferrers and that induces $\mathcal{T}$ on $A \times Z$. This construction nevertheless proves quite useful to deal with the general case, to which we now turn.

\subsection{Biorders: the general case}

The Ferrers property is a necessary condition for the existence of a strict representation (2) or of a non-strict one (1) independently of the cardinality of $A$ and $Z$. Without the restriction that $A$ and $Z$ are countable, order-denseness conditions must be invoked to obtain a numerical representation and, unsurprisingly, the equivalence between strict and non-strict representations does not hold any more. We first tackle the case of a strict representation.

Proposition 2 (Prop. 9, p. 84 in Doignon et al., 1984)

Let $\mathcal{T}$ be a binary relation between $A$ and $Z$. The following statements are equivalent.

1. $\mathcal{T}$ is Ferrers and there is a finite or countably infinite subset $\mathcal{A}^{*} \subseteq A$ such that, for all $a \in A$ and $p \in Z$,

$$
a \mathcal{T} p \Rightarrow\left[a \succsim_{\mathcal{T}}^{\ell} a^{*} \text { and } a^{*} \mathcal{T} p \text {, for some } a^{*} \in \mathcal{A}^{*}\right] .
$$

2. There are a real-valued function $f$ on $A$ and a real-valued function $g$ on $Z$ such that (2) holds.

Furthermore, the functions $f$ and $g$ used in statement 2 can always be chosen so that (3) holds.

\section{Remark 1}

The order-denseness used above is not symmetric between $A$ and $Z$. We use it only to keep things simple. As shown in Doignon et al. (1984, Prop. 8, p. 82), it would be sufficient to require that there is a finite or countably infinite subset of $\mathcal{K}^{*} \subseteq A \cup Z$ such that

$$
a \mathcal{T} p \Rightarrow\left\{\begin{array}{l}
a \succsim_{\mathcal{T}}^{\ell} \alpha^{*} \text { and } \alpha^{*} \mathcal{T} p, \text { for some } \alpha^{*} \in \mathcal{K}^{*} \cap A, \\
a \mathcal{T} \alpha^{*} \text { and } \alpha^{*} \succsim_{\mathcal{T}}^{r} p, \text { for some } \alpha^{*} \in \mathcal{K}^{*} \cap Z .
\end{array}\right.
$$


Nakamura (2002, Th. 3.1) has shown that the order-denseness condition used in Proposition 2 can be equivalently formulated as follows. A pair of subsets $\mathcal{A}^{*} \subseteq A$, $\mathcal{Z}^{*} \subseteq Z$ is said to be jointly dense for $\mathcal{T}$ if, for all $a \in A$ and $p \in Z$,

$$
a \mathcal{T} p \Rightarrow\left[a \succsim_{\mathcal{T}}^{\ell} a^{*}, a^{*} \mathcal{T} p^{*}, p^{*} \succsim_{\mathcal{T}}^{r} p, \text { for some } a^{*} \in \mathcal{A}^{*} \text { and } p^{*} \in \mathcal{Z}^{*}\right]
$$

Nakamura (2002, Th. 3.1) shows that a biorder $\mathcal{T}$ between $A$ and $Z$ has a numerical representation in model (2) iff there are countable subsets $\mathcal{A}^{*} \subseteq A, \mathcal{Z}^{*} \subseteq Z$ that are jointly dense for $\mathcal{T}$.

In order to tackle the non-strict case, it is useful to observe that $\mathcal{T}$ has a non-strict representation iff $\mathcal{T}^{c d}$ has a strict representation.

Proposition 3 (Prop. 8, p. 82 in Doignon et al., 1984)

Let $\mathcal{T}$ be a binary relation between $A$ and $Z$. The following statements are equivalent.

1. $\mathcal{T}$ is Ferrers and there is a finite or countably infinite subset $\mathcal{A}^{*} \subseteq A$ such that, for all $a \in A$ and $p \in Z$,

$$
a \mathcal{T}^{c} p \Rightarrow\left[a^{*} \succsim_{\mathcal{T}}^{\ell} a \text { and } a^{*} \mathcal{T}^{c} p, \text { for some } a^{*} \in \mathcal{A}^{*}\right]
$$

2. There are a real-valued function $f$ on $A$ and a real-valued function $g$ on $Z$ such that (1) holds.

Furthermore, the functions $f$ and $g$ used in statement 2 can always be chosen so that (3) holds.

The sufficiency proof of the above proposition consists in showing that the orderdenseness on $\mathcal{T}^{c}$ implies that there is a countable subset $\mathcal{K}^{*} \subseteq A \cup Z$ that is dense in $A \cup Z$ for the weak order $Q$, i.e., that $\alpha Q^{a} \beta$ implies that $\left[\alpha Q \gamma^{*}\right.$ and $\gamma^{*} Q \beta$, for some $\left.\gamma^{*} \in \mathcal{K}^{*}\right]$.

Again, the order-denseness condition used above is not symmetric between $A$ and $Z$. We refer to Doignon et al. (1984, Prop. 8, p. 82) and to Nakamura (2002, Th. 3.2) for symmetric reformulations of this condition.

\subsection{A lemma on biorders}

We conclude our brief review of biorders by stating a simple lemma that will prove useful in the sequel. 


\section{Lemma 1}

Suppose that $\mathcal{T}$ is a relation between $A$ and $Z$. Let $\succsim_{b}^{\ell}$ be a weak order on $A$ and $\succsim_{b}^{r}$ be a weak order on $Z$. Suppose that, for all $a, b \in A$ and all $p, q \in Z$,

$$
\begin{aligned}
a \succsim_{b}^{\ell} b \text { and } b \mathcal{T} p & \Rightarrow a \mathcal{T} p, \\
p \succsim_{b}^{r} q \text { and } a \mathcal{T} p & \Rightarrow a \mathcal{T} q .
\end{aligned}
$$

Then:

1. $\mathcal{T}$ is a biorder,

2. $\succsim_{b}^{\ell}$ refines $\succsim_{\mathcal{T}}^{\ell}$,

3. $\succsim_{b}^{r}$ refines $\succsim_{\mathcal{T}}^{r}$,

4. the binary relation $\mathscr{Q}$ on $A \cup Z$ that is defined letting, for all $\alpha, \beta \in A \cup Z$,

$$
\alpha \mathscr{Q} \beta \Leftrightarrow\left\{\begin{array}{l}
\alpha \in A, \beta \in A, \text { and } \alpha \succsim_{b}^{\ell} \beta, \\
\alpha \in Z, \beta \in Z, \text { and } \alpha \succsim_{b}^{r} \beta, \\
\alpha \in A, \beta \in Z, \text { and } \alpha \mathcal{T} \beta, \\
\alpha \in Z, \beta \in A, \text { and } \operatorname{Not}[\beta \mathcal{T} \alpha] .
\end{array}\right.
$$

is a weak order.

\section{ProOF}

Part 1. Suppose that $a \mathcal{T} p$ and $b \mathcal{T} q$. Since $\succsim_{b}^{\ell}$ is complete, we have either $b \succsim_{b}^{\ell} a$ or $a \succsim_{b}^{\ell} b$. If $b \succsim_{\tau_{b}^{\ell}} a, a \mathcal{T} p$ implies $b \mathcal{T} p$. In the second case, $b \mathcal{T} q$ implies $a \mathcal{T} q$. Hence, $\mathcal{T}$ is Ferrers.

Part 2. Suppose that $a \succsim_{b}^{\ell} b$ and, in contradiction with the thesis, $b \succ_{\mathcal{T}}^{\ell} a$. This implies that $b \mathcal{T} p$ and $\operatorname{Not}[a \mathcal{T} p]$, for some $p \in Z$. Now, $a \succsim_{b}^{\ell} b$ and $b \mathcal{T} p$ imply $a \mathcal{T} p$, a contradiction. The proof of Part 3 is similar.

Part 4. It is simple to check that $\mathscr{Q}$ is complete. Let us show that it is transitive, i.e., that, for all $\alpha, \beta, \gamma \in A \cup Z, \alpha \mathscr{Q} \beta$ and $\beta \mathscr{Q} \gamma$ imply $\alpha \mathscr{Q} \gamma$. Since each of $\alpha, \beta, \gamma$ can belong either to $A$ or to $Z$, there are 8 cases to examine.

1. If $\alpha, \beta, \gamma \in A$, the conclusion follows from the transitivity of $\succsim_{b}^{\ell}$.

2. If $\alpha, \beta, \gamma \in Z$, the conclusion follows from the transitivity of $\succsim_{b}^{r}$.

3. If $\alpha, \beta \in A$ and $\gamma \in Z, \alpha \mathscr{Q} \beta$ and $\beta \mathscr{Q} \gamma$ means that $\alpha \succsim_{b}^{\ell} \beta$ and $\beta \mathcal{T} \gamma$. This implies $\alpha \mathcal{T} \gamma$, so that $\alpha \mathscr{Q} \gamma$.

4. If $\alpha, \gamma \in A$ and $\beta \in Z, \alpha \mathscr{Q} \beta$ and $\beta \mathscr{Q} \gamma$ means that $\alpha \mathcal{T} \beta$ and $\operatorname{Not}[\gamma \mathcal{T} \beta]$. This implies $\operatorname{Not}\left[\gamma \succsim_{\mathcal{T}}^{\ell} \alpha\right]$. Because $\mathcal{T}$ is a biorder, we know that $\succsim_{\mathcal{T}}^{\ell}$ is complete, so that $\alpha \succ_{\mathcal{T}}^{\ell} \gamma$. Because $\succsim_{b}^{\ell}$ refines $\succsim_{\mathcal{T}}^{\ell}, \alpha \succ_{\mathcal{T}}^{\ell} \gamma$ implies $\alpha \succ_{b}^{\ell} \gamma$, so that $\alpha \mathscr{Q} \gamma$. 
5. If $\beta, \gamma \in A$ and $\alpha \in Z, \alpha \mathscr{Q} \beta$ and $\beta \mathscr{Q} \gamma$ means that $\operatorname{Not}[\beta \mathcal{T} \alpha]$ and $\beta \succsim_{b} \gamma$. Suppose that $\gamma \mathcal{T} \alpha$. Using $\beta \succsim_{b}^{\ell} \gamma$, we obtain $\beta \mathcal{T} \alpha$, a contradiction. Therefore, we must have $\operatorname{Not}[\gamma \mathcal{T} \alpha]$ so that $\alpha \mathscr{Q} \gamma$.

6. If $\alpha, \beta \in Z$ and $\gamma \in A, \alpha \mathscr{Q} \beta$ and $\beta \mathscr{Q} \gamma$ means that $\alpha \succsim_{b}^{r} \beta$ and $\operatorname{Not}[\gamma \mathcal{T} \beta]$. Suppose that $\gamma \mathcal{T} \alpha$. Using $\alpha \succsim_{b}^{r} \beta$, we obtain $\gamma \mathcal{T} \beta$, a contradiction. Hence, we must have $\operatorname{Not}[\gamma \mathcal{T} \alpha]$, so that $\alpha \mathscr{Q} \gamma$.

7. If $\alpha, \gamma \in Z$ and $\beta \in A, \alpha \mathscr{Q} \beta$ and $\beta \mathscr{Q} \gamma$ means that $\operatorname{Not}[\beta \mathcal{T} \alpha]$ and $\beta \mathcal{T} \gamma$. This implies $\operatorname{Not}\left[\gamma \succsim_{\mathcal{T}}^{r} \alpha\right]$. Because $\mathcal{T}$ is a biorder, we know that $\succsim_{\mathcal{T}}^{r}$ is complete, so that $\alpha \succ_{\mathcal{T}}^{r} \gamma$. Because $\succsim_{b}^{r}$ refines $\succsim_{\mathcal{T}}^{r}, \alpha \succ_{\mathcal{T}}^{r} \gamma$ implies $\alpha \succ_{b}^{r} \gamma$, so that $\alpha \mathscr{Q} \gamma$.

8. If $\beta, \gamma \in Z$ and $\alpha \in A, \alpha \mathscr{Q} \beta$ and $\beta \mathscr{Q} \gamma$ means that $\alpha \mathcal{T} \beta$ and $\beta \succsim_{b}^{r} \gamma$. This implies $\alpha \mathcal{T} \gamma$, so that $\alpha \mathscr{Q} \gamma$.

When $\mathcal{T}$ is a biorder, it is clear that the relations $\succsim_{\mathcal{J}}^{\ell}$ and $\succsim_{\mathcal{J}}^{r}$ satisfy the conditions of the above lemma. Note that, since $Q$ is the maximal quasi-order on $A \cup Z$ that coincides with $\mathcal{T}$ on $A \times Z$, the weak order $\mathscr{Q}$ refines $Q$.

\subsection{Interval orders}

An interval order $T$ is an irreflexive Ferrers relation on a set $X$, which implies that it is a biorder between $X$ and $X$. Hence, if $X$ is countable, it has a non-strict numerical representation such that, for all $x, y \in X$,

$$
x T y \Leftrightarrow u(x) \geq v(y),
$$

where $u$ and $v$ are two real-valued function on $X$. Since $T$ is irreflexive, we obtain $u(x)<v(x)$, for all $x \in X$. A similar analysis can be conducted using a strict representation instead of a non-strict one.

This is summarized below, mainly following Fishburn (1970).

\section{Proposition 4}

Let $T$ be a binary relation on a finite or countably infinite set $X$. The following statements are equivalent.

1. $T$ is an interval order

2. There are real-valued functions $u$ and $v$ on $X$ such that, for all $x, y \in X$,

$$
\begin{gathered}
x T y \Leftrightarrow u(x)>v(y), \\
u(x) \leq v(x) .
\end{gathered}
$$


3. There are real-valued functions $u$ and $v$ on $X$ such that, for all $x, y \in X$,

$$
\begin{gathered}
x T y \Leftrightarrow u(x) \geq v(y), \\
u(x)<v(x) .
\end{gathered}
$$

Furthermore, the functions $u$ and $v$ used in statements 2 and 3 can always be chosen so that, for all $x, y \in X$,

$$
\begin{aligned}
& x \succsim_{T}^{\ell} y \Leftrightarrow u(x) \geq u(y), \\
& x \succsim_{T}^{r} y \Leftrightarrow v(x) \geq v(y) .
\end{aligned}
$$

\section{Remark 2}

Using Lemma 1, it is not difficult to check that the above result may be slightly strengthened requiring that the set $X / \sim_{T}$ of equivalence classes of $X$ under $\sim_{T}$ is finite or countably infinite (Fishburn, 1985, Th. 8, p. 29).

Clearly, the order-denseness conditions introduced for biorders are all that is needed to cover the general case. For the record this is stated below for the case of a strict representation. A similar proposition can obviously be obtained for the case of a non-strict representation.

\section{Proposition 5}

Let $T$ be a binary relation on a set $X$. The following statements are equivalent.

1. $T$ is an interval order and there is a finite or countably infinite set $\mathcal{X}^{*} \subseteq X$ such that, for all $x, y \in X$,

$$
x T y \Rightarrow\left[x \succsim_{T}^{\ell} x^{*} \text { and } x^{*} T y, \text { for some } x^{*} \in \mathcal{X}^{*}\right] .
$$

2. There are real-valued functions $u$ and $v$ on $X$ such that (6) holds.

Furthermore, the functions $u$ and $v$ used in statement 2 can always be chosen so that (8) holds.

For a detailed study of the numerical representation of interval orders, we refer to Bosi, Candeal, Induráin, Oloriz, and Zudaire (2001). Early studies of this problem include Bridges (1983a,b, 1985), Doignon et al. (1984), and Fishburn (1970, 1973), Gensemer (1987b), Lück (2004), and Oloriz, Candeal, and Induráin (1998). In model (6), a closed interval $[u(x), v(x)]$ is associated to each element of $X$. A generalization of this representation that allows for intervals that may be closed or open is considered in Fishburn (1985, Ch. 7), and Nakamura (2002). 


\subsection{Semiorders}

A semiorder $T$ is a semitransitive interval order. We envisage below three different forms of numerical representation for such relations, following Aleskerov, Bouyssou, and Monjardet (2007).

\subsubsection{Representations with no proper nesting}

Semitransitivity implies that it is never true that $x \succ_{T}^{\ell} y$ and $y \succ_{T}^{r} x$. When $X$ is countable, this leads to what Aleskerov et al. (2007) called representations with no proper nesting of semiorders, i.e., representations that are identical to the ones envisaged above for interval orders with the added feature that, for all $x, y \in X$,

$$
u(x)>u(y) \Rightarrow v(x) \geq v(y)
$$

This is recorded below.

\section{Proposition 6}

Let $T$ be a binary relation on a finite or countably infinite set $X$. The following statements are equivalent.

1. $T$ is a semiorder.

2. There are real-valued functions $u$ and $v$ on $X$ such that, for all $x, y \in X$,

$$
\begin{gathered}
x T y \Leftrightarrow u(x)>v(y), \\
u(x)>u(y) \Rightarrow v(x) \geq v(y), \\
u(x) \leq v(x) .
\end{gathered}
$$

3. There are real-valued functions $u$ and $v$ on $X$ such that, for all $x, y \in X$,

$$
\begin{gathered}
x T y \Leftrightarrow u(x) \geq v(y), \\
u(x)>u(y) \Rightarrow v(x) \geq v(y), \\
u(x)<v(x) .
\end{gathered}
$$

Furthermore, the functions $u$ and $v$ used in statements 2 and 3 can always be chosen so that (8) holds (Fishburn, 1985, Th. 8, p. 29).

\section{Remark 3}

As with interval orders, the above result may be slightly strengthened requiring that requiring that the set $X / \sim_{T}$ of equivalence classes of $X$ under $\sim_{T}$ is finite or countably infinite. 
The extension to the general case is immediate using the order-denseness conditions introduced above for biorders. The following proposition deals with strict representations in the general case. A similar proposition can be formulated for non-strict representations.

\section{Proposition 7}

Let $T$ be a binary relation on a set $X$. The following statements are equivalent.

1. $T$ is a semiorder and there is a finite or countably infinite set $\mathcal{X}^{*} \subseteq X$ such that (9) holds.

2. There are real-valued functions $u$ and $v$ on $X$ such that (10) holds.

Furthermore, the functions $u$ and $v$ used in statement 2 can always be chosen so that (8) holds.

\subsubsection{Representations with no nesting}

As shown in Fishburn $(1973,1985)$, a more constrained representation can be envisaged for semiorders in which, for all $x, y \in X, u(x) \geq u(y) \Leftrightarrow v(x) \geq v(y)$. Such a representation (called representation with no nesting in Aleskerov et al., 2007), is easy to obtain in the countable case. Its extension to the general case is more delicate (on this point, see Fishburn, 1985, Chap. 7). This is recorded below.

\section{Proposition 8}

Let $T$ be a binary relation on a finite or countably infinite set $X$. The following statements are equivalent.

1. $T$ is a semiorder.

2. There are real-valued functions $u$ and $v$ on $X$ such that, for all $x, y \in X$,

$$
\begin{gathered}
x T y \Leftrightarrow u(x)>v(y), \\
u(x) \geq u(y) \Leftrightarrow v(x) \geq v(y), \\
u(x) \leq v(x) .
\end{gathered}
$$

3. There are real-valued functions $u$ and $v$ on $X$ such that, for all $x, y \in X$,

$$
\begin{gathered}
x T y \Leftrightarrow u(x) \geq v(y), \\
u(x) \geq u(y) \Leftrightarrow v(x) \geq v(y), \\
u(x)<v(x) .
\end{gathered}
$$


Furthermore, the functions $u$ and $v$ used in statements 2 and 3 can always be chosen so that, for all $x, y \in X$,

$$
x \succsim_{T} y \Leftrightarrow u(x) \geq u(y) \Leftrightarrow v(x) \geq v(y) .
$$

\section{ProOF}

It is clear that (12) implies that $T$ is a semiorder. Conversely, we apply Lemma 1 with $\succsim_{b}^{\ell}=\succsim_{T}$ and $\succsim_{b}^{r}=\succsim_{T}$. Because $X$ is at most countable, there is a numerical representation $F$ of the weak order $\mathscr{Q}$ on $X \cup X^{\prime}$, where $X^{\prime}$ is a disjoint duplication of $X$. Defining $u$ (resp. $v$ ) as the restriction of $F$ on $X$ (resp. $X^{\prime}$ ), it is clear that (12) and (14) hold. The proof for (13) is similar once it is observed that if $T$ is Ferrers and semitransitive, the same will be true with $T^{c d}$ and that $\succsim_{T}=\succsim_{T^{c d}}$.

\section{Remark 4}

In the above result, we may only ask that requiring that the set $X / \sim_{T}$ of equivalence classes of $X$ under $\sim_{T}$ is finite or countably infinite.

The following proposition deals with strict representations in the general case. A similar proposition can be formulated for non-strict representations.

Proposition 9 (Fishburn, 1985, Th. 8, p. 136)

Let $T$ be a binary relation on a set $X$. The following statements are equivalent.

1. $T$ is a semiorder and there is a finite or countably infinite set $\mathcal{X}^{*} \subseteq X$ such that (9) holds.

2. There are real-valued functions $u$ and $v$ on $X$ such that (12) holds.

Furthermore, the functions $u$ and $v$ used in statement 2 can always be chosen so that (14) holds.

\subsubsection{Constant threshold representations}

When $X$ is finite, the classic result of Scott and Suppes (1958) shows that it is always possible to obtain a constant threshold representation of semiorders, i.e., a representation in which, for all $x \in X, v(x)=u(x)+1$. It is well-known that constant threshold representations do not extend to the countable case without additional restrictions. The study of constant threshold representations of semiorders on countable sets was pioneered by Manders (1981) and pursued in Beja and Gilboa (1992). The situation is even more complex in the general case as shown in Candeal, Induráin, and Zudaire (2002) and Abrísqueta, Candeal, Induráin, and Zudaire, 2009 (see also Gensemer, 1987a and Narens, 1994). Aleskerov et al. (2007) offer a recent survey of these developments.

The following proposition is proved in Pirlot and Vincke (1997, Th. 3.4) or Fishburn (1985, Th. 2.9, p. 122). 


\section{Proposition 10}

Let $T$ be a binary relation on a finite set $X$. The following statements are equivalent.

1. $T$ is a semiorder

2. There is a real-valued function $u$ such that, for all $x, y \in X$

$$
x T y \Leftrightarrow u(x)>u(y)+1,
$$

3. There is a real-valued function $u$ such that, for all $x, y \in X$

$$
x T y \Leftrightarrow u(x) \geq u(y)+1 .
$$

\section{Biorders with frontier}

\subsection{The model}

Consider now two disjoint relations $\mathcal{T}$ and $\mathcal{F}$ between the sets $A$ and $Z$. We investigate below the conditions on $\mathcal{T}$ and $\mathcal{F}$ under which there are a real-valued function $f$ on $A$ and a real-valued function $g$ on $Z$ such that, for all $a \in A$ and $p \in Z$,

$$
\begin{aligned}
& a \mathcal{T} p \Leftrightarrow f(a)>g(p), \\
& a \mathcal{F} p \Leftrightarrow f(a)=g(p) .
\end{aligned}
$$

The above model constitutes a simple generalization of biorders. Apparently it has never been studied in the literature ${ }^{3}$. We denote by $\mathcal{R}$ the relation between $A$ and $Z$ equal to $\mathcal{T} \cup \mathcal{F}$. Remember from Section 2 that $\mathcal{R}^{c}$ is the relation between $A$ and $Z$ such that $a \mathcal{R}^{c} p \Leftrightarrow \operatorname{Not}[a \mathcal{R} p]$. As above, let $\succsim_{\mathcal{T}}^{\ell}$ (resp. $\succsim_{\mathcal{T}}^{r}$ ) be the trace of $\mathcal{T}$ on $A$ (resp. on $Z$ ). Similarly, let $\succsim_{\mathcal{R}}^{\ell}$ (resp. $\succsim_{\mathcal{R}}^{r}$ ) be the trace of $\mathcal{R}$ on $A$ (resp. on $Z$ ). Define

$$
\succsim_{\star}^{\ell}=\succsim_{\mathcal{T}}^{\ell} \cap \succsim_{\mathcal{R}}^{\ell}
$$

and

$$
\succsim_{\star}^{r}=\succsim_{\mathcal{T}}^{r} \cap \succsim_{\mathcal{R}}^{r}
$$

\footnotetext{
${ }^{3}$ Ducamp and Falmagne (1969, Th. 1, p. 363) have given necessary and sufficient conditions on a relation $\mathcal{F}$ between two finite sets to have a representation $(17 \mathrm{~b})$; their analysis can straightforwardly be extended to cover arbitrary sets. Such relations have been further studied in Doignon and Falmagne (1984) under the name of matching relations.
} 
Hence, we have:

$$
\begin{aligned}
& a \succsim_{\mathcal{T}}^{\ell} b \Leftrightarrow[b \mathcal{T} p \Rightarrow a \mathcal{T} p, \text { for all } p \in Z], \\
& p \succsim_{\mathcal{T}}^{r} q \Leftrightarrow[a \mathcal{T} p \Rightarrow a \mathcal{T} q, \text { for all } a \in A] \\
& a \succsim_{\mathcal{R}}^{\ell} b \Leftrightarrow[b \mathcal{R} p \Rightarrow a \mathcal{R} p, \text { for all } p \in Z], \\
& p \succsim_{\mathcal{R}}^{r} q \Leftrightarrow[a \mathcal{R} p \Rightarrow a \mathcal{R} q, \text { for all } a \in A], \\
& a \succsim_{\star}^{\ell} b \Leftrightarrow[[b \mathcal{T} p \Rightarrow a \mathcal{T} p] \text { and }[b \mathcal{R} p \Rightarrow a \mathcal{R} p], \text { for all } p \in Z], \\
& p \succsim_{\star}^{r} q \Leftrightarrow[[a \mathcal{T} p \Rightarrow a \mathcal{T} q] \text { and }[a \mathcal{R} p \Rightarrow a \mathcal{R} q], \text { for all } a \in A] .
\end{aligned}
$$

By construction, $\succsim_{\mathcal{T}}^{\ell}, \succsim_{\mathcal{R}}^{\ell}, \succsim_{\star}^{\ell}, \succsim_{\mathcal{T}}^{r}, \succsim_{\mathcal{R}}^{r}, \succsim_{\star}^{r}$ are reflexive and transitive. We know that $\succsim_{\mathcal{T}}^{\ell}$ is complete iff $\succsim_{\mathcal{T}}^{r}$ is complete iff $\mathcal{T}$ is a biorder. Similarly, $\succsim_{\mathcal{R}}^{\ell}$ is complete iff $\succsim_{\mathcal{R}}^{r}$ is complete iff $\mathcal{R}$ is a biorder.

Whereas it is clear that the existence of a representation of type (17) implies that $\mathcal{R}$ has a non-strict numerical representation of type (1), the converse is not true as will be shown below (see Lemma 2(2) and the examples following Proposition 12).

\subsection{The countable case}

In view of Proposition 2, we know that (17) implies that both $\mathcal{T}$ and $\mathcal{R}$ are biorders. Clearly, these two biorders must somehow be compatible. This compatibility will be ensured by the two thinness conditions introduced below.

In model (17), the pair of relations $\mathcal{T}$ and $\mathcal{F}$ is "thin" in the following sense. If $a \mathcal{F} p$ and $b \mathcal{F} p$, we have $f(a)=g(p)$ and $f(b)=g(p)$, so that $f(a)=f(b)$. Hence, for all $q \in Z$, we have $a \mathcal{F} q \Leftrightarrow b \mathcal{F} q$ and $a \mathcal{T} q \Leftrightarrow b \mathcal{T} q$. We say that the pair of relations $\mathcal{T}$ and $\mathcal{F}$ is left thin if, for all $a, b \in A$ and $p \in Z$,

$$
[a \mathcal{F} p \text { and } b \mathcal{F} p] \Rightarrow a \sim_{\star}^{\ell} b \text {. }
$$

Similarly, we say that the pair of relations $\mathcal{T}$ and $\mathcal{F}$ is right thin if, for all $a \in A$ and $p, q \in Z$,

$$
[a \mathcal{F} p \text { and } a \mathcal{F} q] \Rightarrow p \sim_{\star}^{r} q
$$

Observe that left thinness (resp. right thinness) may be formulated as $\mathcal{F F}^{d} \subseteq \sim_{\star}^{\ell}$ (resp. $\mathcal{F}^{d} \mathcal{F} \subseteq \sim_{\star}^{\ell}$ ). We say that thinness holds if left and right thinness are satisfied.

Some of the consequences of these conditions are collected below.

\section{Lemma 2}

1. If two disjoint relations $\mathcal{T}$ and $\mathcal{F}$ between the sets $A$ and $Z$ have a representation (17), then $\mathcal{T}$ is a biorder, $\mathcal{R}$ is a biorder, and thinness holds. 
2. For a pair of disjoint relations, the following four conditions are independent: $\mathcal{T}$ is a biorder, $\mathcal{R}$ is a biorder, left thinness holds, right thinness holds.

3. If two disjoint relations $\mathcal{T}$ and $\mathcal{F}$ between $A$ and $Z$ are such that $\mathcal{T}$ and $\mathcal{R}$ are biorders and thinness holds, then the relation $\succsim_{\star}^{\ell}$ on $A$ and the relation $\succsim^{r}$ on $Z$ are both complete.

4. Under the conditions of Part 3, we have:

$$
\begin{aligned}
& {\left[a \mathcal{F} p \text { and } b \succ_{\star}^{\ell} a\right] \Rightarrow b \mathcal{T} p,} \\
& {\left[a \mathcal{F} p \text { and } p \succ_{\star}^{r} q\right] \Rightarrow a \mathcal{T} q,} \\
& {\left[a \mathcal{F} p \text { and } a \succ_{\star}^{\ell} c\right] \Rightarrow c \mathcal{R}^{c} p,} \\
& {\left[a \mathcal{F} p \text { and } r \succ_{\star}^{r} p\right] \Rightarrow a \mathcal{R}^{c} r,}
\end{aligned}
$$

for all $a, b, c \in A$ and $p, q, r \in Z$.

\section{Proof}

\begin{tabular}{|c|c|c|c|}
\hline $\mathcal{T}$ biorder & $\mathcal{R}$ biorder & right thinness & left thinness \\
\hline$q$ & $p$ & $p$ & $p$ \\
\hline $\mathcal{F}$ & $\mathcal{F}$ & $\mathcal{F}$ & $\mathcal{F}$ \\
\hline $\mathcal{F} \quad \mathcal{T}$ & - & $\mathcal{T}$ & $\mathcal{F}$ \\
\hline
\end{tabular}

Part 1 is obvious. The proof of Part 2 follows from the four examples below. We indicate, for each example, which condition among the set of four conditions is the only one to be violated.

Part 3. Suppose that $\succsim_{\star}^{\ell}$ is not complete. Hence, for some $a, b \in A$ and some $p, q \in Z$, we have:

$$
\begin{gathered}
b \mathcal{T} p \text { and } \operatorname{Not}[a \mathcal{T} p], \text { for some } p \in Z, \\
\quad \text { or } \\
b \mathcal{R} p \text { and } \operatorname{Not}[a \mathcal{R} p], \text { for some } p \in Z,
\end{gathered}
$$

and

$$
\begin{gathered}
a \mathcal{T} q \text { and } \operatorname{Not}[b \mathcal{T} q], \text { for some } q \in Z, \\
\text { or } \\
a \mathcal{R} q \text { and } \operatorname{Not}[b \mathcal{R} q], \text { for some } q \in Z,
\end{gathered}
$$

The combination of (19a) and (19c) violates the fact that $\mathcal{T}$ is a biorder. Similarly, the combination of $(19 \mathrm{~b})$ and $(19 \mathrm{~d})$ violates the fact that $\mathcal{R}$ is a biorder. The combination of conditions (19a) and (19d) says that a $\mathcal{R} q, b \mathcal{T} p, \operatorname{Not}[a \mathcal{T} p]$ and $\operatorname{Not}[b \mathcal{R} q]$. Notice that $\operatorname{Not}[a \mathcal{T} p]$ implies either $\operatorname{Not}[a \mathcal{R} p]$ or a $\mathcal{F} p$. If 
$\operatorname{Not}[a \mathcal{R} p]$, since we know that $a \mathcal{R} q, b \mathcal{R} p$ and $\operatorname{Not}[b \mathcal{R} q]$, we have a violation of the fact that $\mathcal{R}$ is a biorder. Hence, we must have $a \mathcal{F} p$. We know that $a \mathcal{R} q$ implies either $a \mathcal{T} q$ or $a \mathcal{F} q$. Suppose that $a \mathcal{T} q$. Since $b \mathcal{T} p$, we obtain, using the fact that $\mathcal{T}$ is a biorder $a \mathcal{T} p$ or $b \mathcal{T} q$, a contradiction. Therefore, we must have $a \mathcal{F} q$. Using right thinness, $a \mathcal{F} p$ and $a \mathcal{F} q$ implies that $b \mathcal{T} p \Leftrightarrow b \mathcal{T} q$, a contradiction. The proof for $\succsim_{\star}^{r}$ is similar.

Part 4. Suppose that $a \mathcal{F} p$ and $b \succ_{\star}^{\ell} a$. Since $a \mathcal{F} p$ implies $a \mathcal{R} p$ and $b \succ_{\star}^{\ell} a$ implies $b \succsim_{\star}^{\ell} a$, we know that $b \mathcal{R} p$. Suppose that $b \mathcal{F} p$. Using left thinness, we know that that $a \mathcal{F} p$ and $b \mathcal{F} p$ imply $b \sim_{\star}^{\ell} a$, a contradiction. Hence, we must have $b \mathcal{T} p$, as required by (18a).

Suppose now that $a \mathcal{F} p$ and $a \succ_{\star}^{\ell} b$ and $b \mathcal{R} p$. If $b \mathcal{F} p, a \mathcal{F} p$ and left thinness imply $a \sim_{\star}^{\ell} b$, a contradiction. Hence, we must have $b \mathcal{T} p$ and $a \succsim^{\ell} b$ implies $b \mathcal{T} p$, a contradiction. This shows that (18c) holds. The proofs of (18b) and (18d) with $\succ_{\star}^{r}$ are similar.

The following lemma generalizes Lemma 1 to cope with a frontier.

\section{Lemma 3}

Let $\mathcal{T}$ and $\mathcal{F}$ be a pair of disjoint relations between $A$ and $Z$. Suppose that $\succsim_{b}^{\ell}$ is a weak order on $A$ and $\succsim_{b}^{r}$ is a weak order on $Z$. Suppose that, for all $\alpha, \beta \in A$ and all $\gamma \in Z$,

$$
\begin{aligned}
& \beta \mathcal{T} \gamma \text { and } \alpha \succsim_{b}^{\ell} \beta \Rightarrow \alpha \mathcal{T} \gamma, \\
& \beta \mathcal{F} \gamma \text { and } \alpha \succ_{b}^{\ell} \beta \Rightarrow \alpha \mathcal{T} \gamma, \\
& \beta \mathcal{F} \gamma \text { and } \alpha \sim_{b}^{\ell} \beta \Rightarrow \alpha \mathcal{F} \gamma .
\end{aligned}
$$

Suppose furthermore that, for all $\alpha \in A$ and all $\beta, \gamma \in Z$,

$$
\begin{aligned}
& \alpha \mathcal{T} \beta \text { and } \beta \succsim_{b}^{r} \gamma \Rightarrow \alpha \mathcal{T} \gamma, \\
& \alpha \mathcal{F} \beta \text { and } \beta \succ_{b}^{r} \gamma \Rightarrow \alpha \mathcal{T} \gamma, \\
& \alpha \mathcal{F} \beta \text { and } \beta \sim_{b}^{r} \gamma \Rightarrow \alpha \mathcal{F} \gamma .
\end{aligned}
$$

Then $\succsim_{b}^{\ell}$ refines $\succsim_{\star}^{\ell}$ and $\succsim_{b}^{r}$ refines $\succsim_{\star}^{r}$. Furthermore, the binary relation $\mathscr{L}$ on $A \cup Z$ that is defined letting, for all $\alpha, \beta \in A \cup Z$,

$$
\alpha \mathscr{L} \beta \Leftrightarrow\left\{\begin{array}{l}
\alpha \in A, \beta \in A, \text { and } \alpha \succsim_{b}^{\ell} \beta, \\
\alpha \in Z, \beta \in Z, \text { and } \alpha \succsim_{b}^{r} \beta, \\
\alpha \in A, \beta \in Z, \text { and } \alpha \mathcal{R} \beta, \\
\alpha \in Z, \beta \in A, \text { and } \operatorname{Not}[\beta \mathcal{T} \alpha] .
\end{array}\right.
$$

is a weak order. 


\section{PROOF}

Let us show that $\succsim_{b}^{\ell}$ refines $\succsim^{\ell}$, the proof that $\succsim_{b}^{r}$ refines $\succsim_{\star}^{r}$ being similar. Suppose that $\alpha \succsim_{b}^{\ell} \beta$, for some $\alpha, \beta \in A$. We have to show that $\alpha \succsim_{\star}^{\ell} \beta$, i.e., that, for all $\gamma \in Z$,

$$
\begin{aligned}
& \beta \mathcal{T} \gamma \Rightarrow \alpha \mathcal{T} \gamma, \\
& \beta \mathcal{R} \gamma \Rightarrow \alpha \mathcal{R} \gamma,
\end{aligned}
$$

Using (20), $\beta \mathcal{T} \gamma$ and $\alpha \succsim_{b}^{\ell} \beta$ imply $\alpha \mathcal{T} \gamma$. Similarly, if $\beta \mathcal{F} \gamma,(20)$ and $\alpha \succsim_{b}^{\ell} \beta$ imply either $\alpha \mathcal{F} \gamma$ or $\alpha \mathcal{T} \gamma$, so that $\alpha \mathcal{R} \gamma$. Hence, $\succsim_{b}^{\ell}$ refines $\succsim_{\star}^{\ell}$.

Let us now show that $\mathscr{L}$ is a weak order. It is simple to check that $\mathscr{L}$ is complete. Let us show that it is transitive, i.e., that, for all $\alpha, \beta, \gamma \in A \cup Z$, $\alpha \mathscr{L} \beta$ and $\beta \mathscr{L} \gamma$ imply $\alpha \mathscr{L} \gamma$. Since each of $\alpha, \beta, \gamma$ can belong either to $A$ or to $Z$, there are 8 cases to examine.

1. If $\alpha, \beta, \gamma \in A$, the conclusion follows from the transitivity of $\succsim_{b}^{\ell}$.

2. If $\alpha, \beta, \gamma \in Z$, the conclusion follows from the transitivity of $\succsim_{b}^{r}$.

3. If $\alpha, \beta \in A$ and $\gamma \in Z, \alpha \mathscr{L} \beta$ and $\beta \mathscr{L} \gamma$ means that $\alpha \succsim_{b}^{\ell} \beta$ and $\beta \mathcal{R} \gamma$. This implies $\alpha \mathcal{R} \gamma$, so that $\alpha \mathscr{L} \gamma$.

4. If $\alpha, \gamma \in A$ and $\beta \in Z, \alpha \mathscr{L} \beta$ and $\beta \mathscr{L} \gamma$ means that $\alpha \mathcal{R} \beta$ and $\operatorname{Not}[\gamma \mathcal{T} \beta]$. Suppose first that $\alpha \mathcal{T} \beta$. Because $\operatorname{Not}[\gamma \mathcal{T} \beta]$, we have $\alpha \succ_{\star}^{\ell} \gamma$. Since $\succsim_{b}^{\ell}$ refines $\succsim_{\star}^{\ell}$, we have $\alpha \succ_{b}^{\ell} \gamma$, so that $\alpha \mathscr{L} \gamma$. Suppose now that $\alpha \mathcal{F} \beta$. Because $\operatorname{Not}[\gamma \mathcal{T} \beta]$, we have either $\gamma \mathcal{F} \beta$ or $\operatorname{Not}[\gamma \mathcal{R} \beta]$. If $\operatorname{Not}[\gamma \mathcal{R} \beta]$, we have $\alpha \succ_{\star}^{\ell} \gamma$, so that $\alpha \succ_{b}^{\ell} \gamma$ and $\alpha \mathscr{L} \gamma$. Suppose now that $\gamma \mathcal{F} \beta$. Because $\alpha \mathcal{F} \beta$, (20) implies that we must have $\alpha \sim_{b}^{\ell} \gamma$, so that $\alpha \mathscr{L} \gamma$.

5. If $\beta, \gamma \in A$ and $\alpha \in Z, \alpha \mathscr{L} \beta$ and $\beta \mathscr{L} \gamma$ means that $N o t[\beta \mathcal{T} \alpha]$ and $\beta \succsim_{b}^{\ell} \gamma$. Suppose that $\gamma \mathcal{T} \alpha$. Using $\beta \succsim_{b}^{\ell} \gamma$, we obtain $\beta \mathcal{T} \alpha$, a contradiction. Therefore, we must have $\operatorname{Not}[\gamma \mathcal{T} \alpha]$ so that $\alpha \mathscr{L} \gamma$.

6. If $\alpha, \beta \in Z$ and $\gamma \in A, \alpha \mathscr{L} \beta$ and $\beta \mathscr{L} \gamma$ means that $\alpha \succsim_{b}^{r} \beta$ and $\operatorname{Not}[\gamma \mathcal{T} \beta]$. Suppose that $\gamma \mathcal{T} \alpha$. Using $\alpha \succsim_{b}^{r} \beta$, we obtain $\gamma \mathcal{T} \beta$, a contradiction. Hence, we must have $\operatorname{Not}[\gamma \mathcal{T} \alpha]$, so that $\alpha \mathscr{L} \gamma$.

7. If $\alpha, \gamma \in Z$ and $\beta \in A, \alpha \mathscr{L} \beta$ and $\beta \mathscr{L} \gamma$ means that $N o t[\beta \mathcal{T} \alpha]$ and $\beta \mathcal{R} \gamma$. Since $\operatorname{Not}[\beta \mathcal{T} \alpha]$, we have either $\beta \mathcal{F} \alpha$ or $\operatorname{Not}[\beta \mathcal{R} \alpha]$. Suppose first that $\operatorname{Not}[\beta \mathcal{R} \alpha]$. Since $\beta \mathcal{R} \gamma$, we have $\alpha \succ_{\star}^{r} \gamma$, so that $\alpha \succ_{b}^{r} \gamma$ and $\alpha \mathscr{L} \gamma$. Suppose now that $\beta \mathcal{F} \alpha$. If $\beta \mathcal{F} \gamma$, then (21) implies that we must have $\alpha \sim_{b}^{r} \gamma$ and $\alpha \mathscr{L} \gamma$. If $\beta \mathcal{T} \gamma$, we have $\alpha \succ_{\star}^{r} \gamma$, so that $\alpha \succ_{b}^{r} \gamma$ and $\alpha \mathscr{L} \gamma$.

8. If $\beta, \gamma \in Z$ and $\alpha \in A, \alpha \mathscr{L} \beta$ and $\beta \mathscr{L} \gamma$ means that $\alpha \mathcal{R} \beta$ and $\beta \succsim_{b}^{r} \gamma$. Because $\succsim_{b}^{r}$ refines $\succsim_{\star}^{r}$, this implies $\alpha \mathcal{R} \gamma$, so that $\alpha \mathscr{L} \gamma$. 
The above lemmas give all what is necessary to obtain the desired numerical representation on countable sets. We have:

Proposition 11

Let $A$ and $Z$ be finite or countably infinite sets and let $\mathcal{T}$ and $\mathcal{F}$ be a pair of disjoint relations between $A$ and $Z$. There are real-valued functions $f$ on $A$ and $g$ on $Z$ such that (17) holds iff $\mathcal{T}$ is a biorder, $\mathcal{R}=\mathcal{T} \cup \mathcal{F}$ is a biorder, and thinness holds. Furthermore, the functions $f$ and $g$ can always be chosen so that, for all $a, b \in A$ and $p, q \in Z$,

$$
\begin{aligned}
& a \succsim_{\star}^{\ell} b \Leftrightarrow f(a) \geq f(b), \\
& p \succsim_{\star}^{r} q \Leftrightarrow g(p) \geq g(q) \text {. }
\end{aligned}
$$

ProOF

Necessity results from Lemma 2(1). Sufficiency is shown applying Lemma 3 with $\succsim_{b}^{\ell}=\succsim_{\star}^{\ell}$ and $\succsim_{b}^{r}=\succsim_{\star}^{r}$. We know that the binary relation $\mathscr{L}$ on $A \cup Z$ is a weak order. Because $A$ and $Z$ are both countable, there is a real-valued function $F$ on $A \cup Z$ such that, for all $\alpha, \beta \in A \cup Z$,

$$
\alpha \mathscr{L} \beta \Leftrightarrow F(\alpha) \geq F(\beta) .
$$

Suppose that, for some, $a \in A$ and $p \in Z$, we have $a \mathcal{T} p$. This implies $a \mathscr{L} p$ and $\operatorname{Not}[p \mathscr{L} a]$ so that $F(a)>F(p)$. Similarly $a \mathcal{F} p$ implies both of $a \mathscr{L} p$ and $p \mathscr{L} a$, so that $F(a)=F(p)$. If $\operatorname{Not}[a \mathcal{R} b]$ we have $\operatorname{Not}[a \mathscr{L} p]$ and $p \mathscr{L} a$, so that $F(a)<F(p)$. Therefore defining $f$ (resp. $g$ ) to be the restriction of $F$ on $A$ (resp. $Z$ ) leads to a representation satisfying (17). In view of the definition of $\mathscr{L}$, it is clear that (22) will hold.

\subsection{The general case}

The extension of Proposition 11 calls for the introduction of order-denseness conditions. We say that a subset $\mathcal{A}^{*} \subseteq A$ is dense for the pair $\mathcal{T}$ and $\mathcal{F}$ if, for all $a \in A$ and all $p \in Z$,

$$
\begin{aligned}
a \mathcal{T} p & \Rightarrow\left[a \succsim_{\star}^{\ell} a^{*} \text { and } a^{*} \mathcal{T} p, \text { for some } a^{*} \in \mathcal{A}^{*}\right], \\
a \mathcal{R}^{c} p & \Rightarrow\left[a^{*} \mathcal{R}^{c} p \text { and } a^{*} \succsim_{\star}^{\ell} a, \text { for some } a^{*} \in \mathcal{A}^{*}\right] .
\end{aligned}
$$

The intuition behind these conditions should be clear. Condition (17a) imposes that the relation $\mathcal{T}$ has a strict representation. Hence, we must impose on $\mathcal{T}$ the order-denseness condition used in Proposition 2. This is exactly what (23) does. Similarly, the conjunction of conditions (17a) and (17b) imposes that the relation $\mathcal{R}$ has a non-strict representation. Hence, we must require that $\mathcal{R}$ satisfies the order-denseness condition used in Proposition 3. This is exactly what (24) does.

The existence of finite or countably infinite subset $\mathcal{A}^{*}$ that is dense for the pair $\mathcal{T}$ and $\mathcal{F}$ will guarantee the existence of numerical representation. We have: 


\section{Proposition 12}

Let $A$ and $Z$ be two sets and let $\mathcal{T}$ and $\mathcal{F}$ be a pair of disjoint relations between $A$ and $Z$. There are real-valued functions $f$ on $A$ and $g$ on $Z$ such that (17) holds iff $\mathcal{T}$ is a biorder, $\mathcal{R}=\mathcal{T} \cup \mathcal{F}$ is a biorder, thinness holds, and there is a finite or countably infinite subset $\mathcal{A}^{*} \subseteq A$ that is dense for the pair $\mathcal{T}$ and $\mathcal{F}$. Furthermore, the functions $f$ and $g$ can always be chosen so that (22) holds.

\section{ProOF}

Necessity. Suppose that there are real-valued functions $f$ on $A$ and $g$ on $Z$ such that (17) holds. Let us show that this implies the existence of a finite or countably infinite subset $\mathcal{A}^{*} \subseteq A$ that is dense for the pair of disjoint relations $\mathcal{T}$ and $\mathcal{F}$.

Let $\lambda_{j} \in f(A)$ be such that

$$
\mu_{j}<\lambda_{j} \text { and }\left(\mu_{j}, \lambda_{j}\right) \cap f(A)=\varnothing,
$$

for some $\mu_{j} \in g(Z)$. With each such $\lambda_{j} \in f(A)$, we associate a particular $\mu_{j} \in g(Z)$ such that (25) holds. Suppose that $\lambda_{k}<\lambda_{j}$. The two intervals $\left(\mu_{k}, \lambda_{k}\right)$ and $\left(\mu_{j}, \lambda_{j}\right)$ are disjoint since $\lambda_{k}>\mu_{j}$ would violate the fact that $\left(\mu_{j}, \lambda_{j}\right) \cap f(A)=\varnothing$. The collection of the numbers $\lambda_{j}$ must be countable because the intervals $\left(\mu_{j}, \lambda_{j}\right)$ are nonempty and disjoint and, therefore, each one contains a distinct rational number. Therefore, there is a finite or countably infinite set $\mathcal{A}_{1}^{*} \subseteq A$ such that $f\left(\mathcal{A}_{1}^{*}\right)$ contains all the $\lambda_{j}$.

Let $\lambda_{j} \in f(A)$ be such that

$$
\lambda_{j}<\mu_{j} \text { and }\left(\lambda_{j}, \mu_{j}\right) \cap f(A)=\varnothing,
$$

for some $\mu_{j} \in g(Z)$. With each such $\lambda_{j} \in f(A)$, we associate a particular $\mu_{j} \in g(Z)$ such that (26) holds. Suppose that $\lambda_{j}<\lambda_{k}$. The two intervals $\left(\lambda_{k}, \mu_{k}\right)$ and $\left(\lambda_{j}, \mu_{j}\right)$ are disjoint since $\lambda_{k}<\mu_{j}$ would violate the fact that $\left(\lambda_{j}, \mu_{j}\right) \cap f(A)=\varnothing$. The collection of the numbers $\lambda_{j}$ must be countable because the intervals $\left(\lambda_{j}, \mu_{j}\right)$ are nonempty and disjoint and, therefore, each one contains a distinct rational number. Therefore, there is a finite or countably infinite set $\mathcal{A}_{2}^{*} \subseteq A$ such that $f\left(\mathcal{A}_{2}^{*}\right)$ contains all the $\lambda_{j}$.

Let us select a subset $\mathcal{A}_{3}^{*} \subseteq A$ such that for every pair of rational numbers $p$ and $q$ such that $p<q$ the following condition holds:

$$
(p, q) \cap f(A) \neq \varnothing \Rightarrow\left[p<f\left(a^{*}\right)<q \text {, for some } a^{*} \in \mathcal{A}_{3}^{*}\right] .
$$

The set $\mathcal{A}_{3}^{*} \subseteq A$ can always be taken to be finite or countably infinite.

Define $\mathcal{A}^{*}=\mathcal{A}_{1}^{*} \cup \mathcal{A}_{2}^{*} \cup \mathcal{A}_{3}^{*}$. By construction, $\mathcal{A}^{*} \subseteq A$ is finite or countably infinite. Let us show that $\mathcal{A}^{*}$ is dense for the pair $\mathcal{T}$ and $\mathcal{F}$.

Suppose that $a \mathcal{T} p$, so that $f(a)>g(p)$. If $(g(p), f(a)) \cap f(A)=\varnothing$ then, by construction, we have $f(a)=f\left(a^{*}\right)$, for some $a^{*} \in \mathcal{A}_{1}^{*}$. Because $f(a)=f\left(a^{*}\right)>$ 
$g(p)$, we clearly have $a \succsim^{\ell} a^{*}$ and $a^{*} \mathcal{T} p$. Otherwise we have $(g(p), f(a)) \cap f(A) \neq$ $\varnothing$ and let $c$ be any element in $A$ such that $g(p)<f(c)<f(a)$. Let $p, q \in \mathbb{Q}$ be such that $g(p)<p<f(c)<q<f(a)$. By construction of the set $\mathcal{A}_{3}^{*}$, we have $g(p)<p<f\left(a^{*}\right)<q<f(a)$, for some $a^{*} \in \mathcal{A}_{3}^{*}$. Because $f(a)>f\left(a^{*}\right)>g(p)$, we have $a^{*} \mathcal{T} p$ and $a \succsim_{\star}^{\ell} a^{*}$.

Suppose now that $a \mathcal{R}^{c} p$, so that $f(a)<g(b)$. If $(f(a), g(p)) \cap f(A)=\varnothing$, then, by construction, we have $f(a)=f\left(a^{*}\right)$, for some $a^{*} \in \mathcal{A}_{2}^{*}$. Because $f(a)=f\left(a^{*}\right)<$ $g(p)$, we have $a^{*} \mathcal{R}^{c} p$ and $a^{*} \succsim_{\star}^{\ell} a$. Otherwise we have $(f(a), g(p)) \cap f(A) \neq \varnothing$ and let $d$ be any element in $A$ such that $f(a)<f(d)<g(p)$. Let $p, q \in \mathbb{Q}$ be such that $f(a)<p<f(d)<q<g(p)$. By construction of the set $\mathcal{A}_{3}^{*}$, we have $f(a)<p<f\left(a^{*}\right)<q<g(p)$, for some $a^{*} \in \mathcal{A}_{3}^{*}$. Because $f(a)<f\left(a^{*}\right)<g(p)$, we have $a^{*} \mathcal{R}^{c} p$ and $a^{*} \succsim_{\star}^{\ell} a$.

Sufficiency. Define the relation $Q_{\star}$ on $A \cup Z$ as the relation $\mathscr{L}$ defined in Lemma 3 with $\succsim_{b}^{\ell}=\succsim_{\star}^{\ell}$ and $\succsim_{b}^{r}=\succsim_{\star}^{r}$. The proof will be complete if we show that there is a countable subset $\mathcal{K}^{*}$ of $A \cup Z$ that is dense for $Q_{\star}$, i.e., that, for all $\alpha, \beta \in A \cup Z$,

$$
\alpha Q_{\star}^{a} \beta \Rightarrow\left[\alpha Q_{\star} \gamma \text { and } \gamma Q_{\star} \beta, \text { for some } \gamma \in \mathcal{K}^{*}\right]
$$

By hypothesis, we know that there is a finite or countably infinite subset $\mathcal{A}^{*} \subseteq A$ that is dense for the pair $\mathcal{T}$ and $\mathcal{F}$. Let us show that this set $\mathcal{A}^{*}$ is dense for the weak order $Q_{\star}$. There are four cases to consider.

1. Suppose that $\alpha \in A$ and $\beta \in Z$. Then $\alpha Q_{\star} \beta$ and $\operatorname{Not}\left[\beta Q_{\star} \alpha\right]$ implies $\alpha \mathcal{T} \beta$. Using the fact that $\mathcal{A}^{*} \subseteq A$ is dense for the pair $\mathcal{T}$ and $\mathcal{F}$, we obtain $\alpha \succsim_{\star}^{\ell} a^{*}$ and $a^{*} \mathcal{T} \beta$, for some $a^{*} \in \mathcal{A}^{*}$, so that $\alpha Q_{\star} a^{*}$ and $a^{*} Q_{\star} \beta$.

2. Suppose that $\alpha \in Z$ and $\beta \in A$. Then $\alpha Q_{\star} \beta$ and $\operatorname{Not}\left[\beta Q_{\star} \alpha\right]$ implies $\beta \mathcal{R}^{c} \alpha$. Using the fact that $\mathcal{A}^{*} \subseteq A$ is dense for the pair $\mathcal{T}$ and $\mathcal{F}$, we obtain $a^{*} \succsim_{\star}^{\ell} \beta$ and $a^{*} \mathcal{R}^{c} \alpha$, for some $a^{*} \in \mathcal{A}^{*}$, so that $\alpha Q_{\star} a^{*}$ and $a^{*} Q_{\star} \beta$.

3. Suppose that $\alpha, \beta \in A$, so that $\alpha Q_{\star} \beta$ and $\operatorname{Not}\left[\beta Q_{\star} \alpha\right]$ implies $\alpha \succ_{\star}^{\ell} \beta$. By definition, we have either

$$
\begin{aligned}
& \alpha \mathcal{T} p \text { and } \beta \mathcal{T}^{c} p, \\
& \text { or } \\
& \alpha \mathcal{R} p \text { and } \beta \mathcal{R}^{c} p,
\end{aligned}
$$

for some $p \in Z$.

Suppose that (28a) holds. Using the fact that $\mathcal{A}^{*} \subseteq A$ is dense for the pair $\mathcal{T}$ and $\mathcal{F}, \alpha \mathcal{T} p$ implies $\alpha \succsim_{\star}^{\ell} a^{*}$ and $a^{*} \mathcal{T} p$, for some $a^{*} \in \mathcal{A}^{*}$. If $\beta \succsim_{\star}^{\ell} a^{*}$, $a^{*} \mathcal{T} p$ implies $\beta \mathcal{T} p$, a contradiction. Hence, we must have $a^{*} \succ_{\star}^{\ell} \beta$, so that 
$\alpha \succsim_{\star}^{\ell} a^{*}$ and $a^{*} \succ_{\star}^{\ell} \beta$. We therefore have $\alpha Q_{\star} a^{*}$ and $a^{*} Q_{\star} \beta$, for some $a^{*} \in \mathcal{A}^{*}$, as required.

Suppose now that $(28 \mathrm{~b})$ holds. Using the fact that $\mathcal{A}^{*} \subseteq A$ is dense for the pair $\mathcal{T}$ and $\mathcal{F}, \beta \mathcal{R}^{c} p$ implies $a^{*} \mathcal{R}^{c} p$ and $a^{*} \succsim^{\ell} \beta$, for some $a^{*} \in \mathcal{A}^{*}$. If $a^{*} \succsim_{\star}^{\ell} \alpha, \alpha \mathcal{R} p$ implies $a^{*} \mathcal{R} p$, a contradiction. Hence, we must have $\alpha \succ_{\star}^{\ell} a^{*}$, so that $\alpha \succ_{\star}^{\ell} a^{*}$ and $a^{*} \succsim_{\star}^{\ell} \beta$. We therefore have $\alpha Q_{\star} a^{*}$ and $a^{*} Q_{\star} \beta$, for some $a^{*} \in \mathcal{A}^{*}$, as required.

4. Suppose that $\alpha, \beta \in Z$, so that $\alpha Q_{\star} \beta$ and $N o t\left[\beta Q_{\star} \alpha\right]$ implies $\alpha \succ_{\star}^{r} \beta$. By definition, we have either

$$
a \mathcal{T} \beta \text { and } a \mathcal{T}^{c} \alpha,
$$

or

$$
a \mathcal{R} \beta \text { and } a \mathcal{R}^{c} \alpha
$$

for some $a \in A$.

Suppose that (28c) holds. Using the fact that $\mathcal{A}^{*} \subseteq A$ is dense for the pair $\mathcal{T}$ and $\mathcal{F}, a \mathcal{T} \beta$ implies $a \succsim_{\star}^{\ell} a^{*}$ and $a^{*} \mathcal{T} \beta$, for some $a^{*} \in \mathcal{A}^{*}$. If $a^{*} \mathcal{T} \alpha$, $a \succsim_{\star}^{\ell} a^{*}$ implies $a \mathcal{T} \alpha$, a contradiction. Therefore, we must have $\operatorname{Not}\left[a^{*} \mathcal{T} \alpha\right]$. Hence, we have $\operatorname{Not}\left[a^{*} \mathcal{T} \alpha\right]$ and $a^{*} \mathcal{R} \beta$, so that $\alpha Q_{\star} a^{*}$ and $a^{*} Q_{\star} \beta$, as required.

Suppose finally that (28d) holds. Using the fact that $\mathcal{A}^{*} \subseteq A$ is dense for the pair $\mathcal{T}$ and $\mathcal{F}, a \mathcal{R}^{c} \alpha$ implies $a^{*} \mathcal{R}^{c} \alpha$ and $a^{*} \succsim_{\star}^{\ell} a$, for some $a^{*} \in \mathcal{A}^{*}$. If $a^{*} \mathcal{R}^{c} \beta, a^{*} \succsim^{\ell} a$ implies $a \mathcal{R}^{c} \beta$, a contradiction. Therefore, we must have $a^{*} \mathcal{R} \beta$. Hence, we have $a^{*} \mathcal{R}^{c} \alpha$ and $a^{*} \mathcal{R} \beta$, so that $a Q_{\star} a^{*}$ and $a^{*} Q_{\star} \beta$, as required.

The above result uses six conditions: the four conditions used in Proposition 11 $(\mathcal{T}$ is a biorder, $\mathcal{R}=\mathcal{T} \cup \mathcal{F}$ is a biorder, left thinness, right thinness, and the two order-denseness conditions (23) and (24). Let us show that these six conditions are independent. It is clear that when both $A$ and $Z$ are countable, the two orderdenseness conditions are trivially satisfied. The examples presented in the proof of Lemma 2(2) can therefore be used to deal with the first four conditions. Let $A$ and $Z$ be disjoint duplications of $\mathbb{R}$ and let $\mathcal{T}$ be such that (abusing notation in an obvious way) $a \mathcal{T} x \Leftrightarrow a>x$. Suppose furthermore that $\mathcal{F}$ is empty. It is clear that $\mathcal{T}$ is a biorder and that the same is true for $\mathcal{R}$. The two thinness conditions are trivially satisfied. Since $\mathcal{T}$ has a strict numerical representation, condition (23) holds. Condition (24) is violated since $\mathcal{R}$ cannot have a non-strict representation. Modifying the last example by taking $a \mathcal{T} x \Leftrightarrow a \geq x$ clearly leads to a situation in which all conditions used in Proposition 12 are satisfied, except (23). 
We briefly examine below how the above results may be used to tackle the case of interval orders and semiorders with frontier. The application to interval orders will be straightforward. The case of semiorders will be slightly more delicate.

\section{$5 \quad$ Interval orders with frontier}

Let $T$ and $F$ be two disjoint relations on $X$. Define $R=T \cup F$.

We are interested in conditions ensuring the existence of two real-valued functions $u$ and $v$ on $X$ such that, for all $x, y \in X$,

$$
\begin{gathered}
x T y \Leftrightarrow u(x)>v(y), \\
x F y \Leftrightarrow u(x)=v(y), \\
u(x)<v(x) .
\end{gathered}
$$

A pair of relations $T$ and $F$ having such a representation is called an interval order with frontier.

Model (29) implies that $u<v$, so that that both $T$ and $F$ must be irreflexive ${ }^{4}$. Using Lemma 2(1), we know that model (29) implies that both $T$ and $R$ must be Ferrers (notice that, because both $T$ and $F$ are irreflexive, this will imply that $T$ and $R$ are asymmetric). Hence, a necessary condition for model (29) is that both $T$ and $R$ are interval orders.

Remember from Section 3 that we have defined $\succsim_{\star}^{\ell}$ on $X$ as $\succsim_{T}^{\ell} \cap \succsim_{R}^{\ell}$ and $\succsim_{\star}^{r}$ on $X$ as $\succsim_{T}^{r} \cap \succsim_{R}^{r}$.

It is easy to check that (29) implies left and right thinness. Indeed, suppose that $x F z$, and $y F z$. This implies $u(x)=v(z)$ and $u(x)=v(z)$, so that $u(x)=u(y)$. Hence, we must have $x F w \Leftrightarrow y F w$ and $x T w \Leftrightarrow y T w$, for all $w \in X$. A similar reasoning shows the necessity of the right thinness condition.

It is straightforward to adapt the examples given above for biorders with frontier to show that the following four conditions are independent: $T$ is an interval order, $R$ is an interval order, left thinness, and right thinness. Using Lemma 2(3), it is simple to check that when both $T$ and $R$ are interval orders, and thinness holds, both relations $\succsim_{\star}^{\ell}$ and $\succsim_{\star}^{r}$ are complete.

This leads to the following corollary of Proposition 11.

\section{Proposition 13}

Let $X$ be a finite or countably infinite set. Let $T$ and $F$ be a pair of disjoint relations on $X$ and define $R=T \cup F$. There are real-valued functions $u$ and $v$ on $X$ such that (29) holds iff $T$ is an interval order, $R$ is an interval order, and

\footnotetext{
${ }^{4}$ Note that if $F$ were supposed to be reflexive, model (29) would be identical to the classic numerical representation of a weak order $\succsim$ with $T=\succ$ and $F=\sim$.
} 
thinness holds. Furthermore, the functions $u$ and $v$ can always be chosen so that, for all $x, y \in X$,

$$
\begin{aligned}
& x \succsim_{\star}^{\ell} y \Leftrightarrow u(x) \geq u(y), \\
& x \succsim_{\star}^{r} y \Leftrightarrow v(x) \geq v(y) \text {. }
\end{aligned}
$$

\section{ProOF}

Necessity was noted above. Sufficiency is established noting that the conditions imposed on $T$ and $F$ imply the conditions used in Proposition 11. The fact that $u<v$ follows from the irreflexivity of $R$.

It suffices to bring the order-denseness condition used in Proposition 12 into the picture to be able to cover the general case. This is recorded below.

\section{Proposition 14}

Let $T$ and $F$ be a pair of disjoint relations on $X$ and define $R=T \cup F$. There are real-valued functions $u$ and $v$ on $X$ such that (29) holds iff $T$ is an interval order, $R$ is an interval order, thinness holds, and there is a countable subset $\mathcal{X}^{*} \subseteq X$ such that, for all $x, y \in X$,

$$
\begin{aligned}
x T y & \Rightarrow\left[x \succsim_{\star}^{\ell} x^{*} \text { and } x^{*} T y\right], \\
x R^{c} y & \Rightarrow\left[x^{*} R^{c} y \text { and } x^{*} \succsim_{\star}^{\ell} x\right],
\end{aligned}
$$

for some $x^{*} \in \mathcal{X}^{*}$. Furthermore, the functions $u$ and $v$ can always be chosen so that (30) holds.

We now turn to the more delicate case of semiorders with frontier.

\section{Semiorders with frontier}

\subsection{Representations with no proper nesting}

We are first interested in conditions that will ensure the existence of two real-valued functions $u$ and $v$ on $X$ such that, $x, y \in X$,

$$
\begin{gathered}
x T y \Leftrightarrow u(x)>v(y), \\
x F y \Leftrightarrow u(x)=v(y), \\
u(x)>u(y) \Rightarrow v(x) \geq v(y), \\
u(x)<v(x),
\end{gathered}
$$

which specializes the representation envisaged above for interval orders with frontier by adding a consistency condition between $u$ and $v$. 
It is clear that model (33) implies that both $T$ and $R$ must be semiorders. It also implies that

$$
\begin{aligned}
& T F R^{s c} \subseteq T, \\
& R^{s c} F T \subseteq T .
\end{aligned}
$$

Indeed, if $a T b, b F c$ and $c R^{s c} d$, we have $u(a)>v(b), u(b)=v(c), u(c)<v(d)$, and $u(d)<v(c)$. In contradiction with the thesis, suppose that $\operatorname{Not}[a T d]$, so that $v(d) \geq u(a)$. Because $v(d) \geq u(a)>v(b)$, we obtain $v(d)>v(b)$. Since $u(b)=v(c)>u(d)$, we have $u(b)>u(d)$, contradicting the fact that $u(x)>$ $u(y) \Rightarrow v(x) \geq v(y)$. The proof that $R^{s c} F T \subseteq T$ holds is similar.

Conditions (34) are inspired from the study of pseudo-orders in Vincke (1980) and Roy and Vincke (1987). In pseudo-orders, the frontier $F$ between $T$ and $R^{s c}$ does not have to be "thin". Model (33) being a particular case of model (29), it implies both left and right thinness.

\section{Lemma 4}

For a pair of disjoint irreflexive relations $T$ and $F$, the following conditions are independent: $T$ is Ferrers, $T$ is semitransitive, $R$ is Ferrers, $R$ is semitransitive, $T F R^{s c} \subseteq T, R^{s c} F T \subseteq T$, left thinness, and right thinness.

\section{ProOF}

We provide the eight required examples indicating, for each example, which condition is the only one to be violated. The first six are taken from Roy and Vincke (1987, page 267), with a minor adaptation for the second one.

$$
\begin{aligned}
& R \text { Ferrers } \quad R \text { semi-transitive }
\end{aligned}
$$

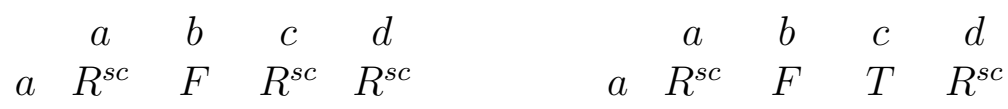

$$
\begin{aligned}
& b-R^{s c} R^{s c} R^{s c} \quad b \quad-R^{s c} F R^{s c} \\
& c-\quad-R^{s c} F \quad c--R^{s c} R^{s c} \\
& d-\quad-\quad R^{s c} \quad d-\quad-\quad-R^{s c} \\
& T \text { Ferrers } \\
& \begin{array}{ccccc} 
& a & b & c & d \\
a & R^{s c} & T & R^{s c} & F
\end{array} \\
& b-R^{s c}-R^{s c} \\
& c-F \quad R^{s c} T \\
& d-\quad-\quad R^{s c} \\
& T F R^{s c} \subseteq T \\
& \begin{array}{ccccc} 
& a & b & c & d \\
a & R^{s c} & T & T & F
\end{array} \\
& b-R^{s c} F R^{s c} \\
& c-\quad-R^{s c} R^{s c} \\
& d-\quad-\quad R^{s c} \\
& T \text { semi-transitive } \\
& \begin{array}{ccccc} 
& a & b & c & d \\
a & R^{s c} & T & T & F
\end{array} \\
& b-R^{s c} T R^{s c} \\
& c-\quad-R^{s c}- \\
& d-\quad-F R^{s c} \\
& R^{s c} F T \subseteq T \\
& \begin{array}{ccccc} 
& a & b & c & d \\
a & R^{s c} & R^{s c} & R^{s c} & F
\end{array} \\
& b-R^{s c} F \quad T \\
& c-\quad-R^{s c} T \\
& d-\quad-\quad R^{s c}
\end{aligned}
$$




\begin{tabular}{|c|c|c|c|c|c|c|c|c|}
\hline \multicolumn{4}{|c|}{ right thinness } & & \multicolumn{4}{|c|}{ left thinness } \\
\hline$a$ & $b$ & $c$ & $d$ & & $a$ & $b$ & $c$ & $d$ \\
\hline$R^{s c}$ & $R^{s c}$ & $F$ & $F$ & $a$ & $R^{s c}$ & $R^{s c}$ & $T$ & $F$ \\
\hline- & $R^{s c}$ & $R^{s c}$ & $T$ & $b$ & - & $R^{s c}$ & $R^{s c}$ & $F$ \\
\hline- & - & $R^{s c}$ & $R^{s c}$ & $c$ & - & - & $R^{s c}$ & $R^{s c}$ \\
\hline- & - & - & $R^{s c}$ & $d$ & - & - & - & $R^{s c}$ \\
\hline
\end{tabular}

We define the trace of the pair of relations $T$ and $F$ as the binary relation $\succsim \star$ on $X$ such that, for all $x, y \in X$,

$$
x \succsim_{\star} y \Leftrightarrow\left[x \succsim_{\star}^{\ell} y \text { and } x \succsim_{\star}^{r} y\right]
$$

By construction, $\succsim_{\star}$ is reflexive and transitive. As shown below, the conditions introduced so far imply that the relation $\succsim_{\star}$ is also complete.

\section{Lemma 5}

Let $T$ and $F$ be a pair of disjoint relations on $X$. If $T$ is a semiorder, $R$ is a semiorder, $T F R^{s c} \subseteq T, R^{s c} F T \subseteq T$, and thinness holds, then ${ }_{\star}$ is complete.

PROOF

Let us show that $\succsim_{\star}$ is complete. Using the results in Vincke (1980) or in Roy and Vincke (1987), it suffices to prove that we have $F R^{s c} T \subseteq T$ and $T R^{s c} F \subseteq T$. Suppose that $a F b, b R^{s c} c$ and $c T d$. Using the fact that $R$ is a semiorder, we must have $a R d$. Suppose that $a F d$. Using right thinness, $a F b, a F d$, and $c T d$ imply $c T b$, a contradiction. Hence, we must have $a T d$, so that $F R^{s c} T \subseteq T$. Suppose now that $a T b, b R^{s c} c$ and $c F d$. Using the fact that $R$ is a semiorder, we must have $a R d$. Suppose that a $F d$. Using left thinness, $c F d$, $a F d$, and $a T b$ imply $c T b$, a contradiction. Hence, we must have $a T d$, so that $T R^{s c} F \subseteq T$.

This leads to our first result representations with no proper nesting.

\section{Proposition 15}

Let $T$ and $F$ be a pair of disjoint relations on a finite or countably infinite set $X$. Define $R=T \cup F$. There are real-valued functions $u$ and $v$ on $X$ such that (33) holds iff $T$ is a semiorder, $R$ is a semiorder, TF $R^{s c} \subseteq T, R^{s c} F T \subseteq T$, and thinness holds. Furthermore the functions $u$ and $v$ can always be chosen so that (30) holds.

\section{PROOF}

Necessity was shown above. Sufficiency is established as in the proof of the Proposition 13. The only difference is that we must show that $u(x)>u(y) \Rightarrow v(x) \geq v(y)$. We know that $u$ is a representation of $\succsim_{\star}^{\ell}$ and that $v$ is a representation of $\succsim_{\star}^{r}$. Lemma 5 has shown that $\succsim_{\star}=\succsim_{\star}^{\ell} \cap \succsim^{r}$ is complete. Hence it is impossible to have $x \succ_{\star}^{\ell} y$ and $y \succ_{\star}^{r} x$. This completes the proof. 
Adding the two order-denseness conditions used in Proposition 12, allows to deal with the general case.

\section{Proposition 16}

Let $T$ and $F$ be a pair of disjoint relations on $X$. Define $R=T \cup F$. There are real-valued functions $u$ and $v$ on $X$ such that (33) holds iff $T$ is a semiorder, $R$ is a semiorder, $T F R^{s c} \subseteq T, R^{s c} F T \subseteq T$, thinness holds, and there is a countable subset $\mathcal{X}^{*} \subseteq X$ such that (31) and (32) hold. Furthermore, the functions $u$ and $v$ can always be chosen so that (30) holds.

\subsection{Representations with no nesting}

A representation with no nesting is such that there are real-valued functions $u$ and $v$ on $X$ such that, for all $x, y \in X$,

$$
\begin{gathered}
x T y \Leftrightarrow u(x)>v(y), \\
x F y \Leftrightarrow u(x)=v(y), \\
u(x) \geq u(y) \Leftrightarrow v(x) \geq v(y), \\
u(x)<v(x) .
\end{gathered}
$$

For semiorders without frontier, we know that representations with no proper nesting are equivalent to representations with no nesting. With the introduction of frontier, this equivalence fails. This leads to study a structure that is more constrained that the one envisaged in the preceding section.

All conditions needed for the existence of a representation with no proper nesting are clearly necessary for model (36). However, model (36) implies a stronger form of thinness that the one required above. Suppose indeed that $x F z$ and $y F z$. This implies $u(x)=v(z)$ and $u(y)=v(z)$, so that $u(x)=u(y)$. This implies that $v(x)=v(y)$. Hence, it will impossible to distinguish $x$ and $y$. A similar conclusion clearly holds if we have $z F x$ and $z F y$. This is the motivation for the following two conditions.

We say the pair of relations $T$ and $F$ is strongly left thin if, for all $x, y, z \in X$, $[x F z$ and $y F z]$ implies that $x \sim_{\star} y$. Similarly, we say that the pair of relations $T$ and $F$ is strongly right thin if, for all $x, y, z \in X,[z F x$ and $z F y]$ implies that $x \sim_{\star} y$. Observe that strong left and right thinness may equivalently be formulated as $F F^{d} \subseteq \sim_{\star}$ and $F^{d} F \subseteq \sim_{\star}$. We say that strong thinness holds if left and right strong thinness are satisfied.

The difference between strong thinness and thinness is illustrated below. Let 
$X=\{a, b, c, d\}$ and let $T$ and $F$ be defined as follows:

$$
\begin{array}{ccccc} 
& a & b & c & d \\
a & R^{s c} & R^{s c} & T & T \\
b & - & R^{s c} & R^{s c} & F \\
c & - & - & R^{s c} & F \\
d & - & - & - & R^{s c}
\end{array}
$$

The pair of relations $T$ and $F$ is thin. It has a representation with no proper nesting taking, e.g., $v(a)=4, u(a)=3, v(b)=4, u(b)=1, v(c)=2, u(c)=1$, and $v(d)=1, u(d)=0$. It cannot have a representation without nesting since strong left thinness is violated. Indeed, $b F d$ and $c F d$ imply $u(b)=u(c)$. In a representation with no nesting, we must also have $v(b)=v(c)$. This violates the fact that $a R^{s c} b$ while $a T c$.

Replacing thinness by strong thinness does not alter the independence of the conditions used in Proposition 15.

\section{Lemma 6}

For a pair of disjoint irreflexive relations $T$ and $F$, the following conditions are independent: $T$ is Ferrers, $T$ is semitransitive, $R$ is Ferrers, $R$ is semitransitive, $T F R^{s c} \subseteq T, R^{s c} F T \subseteq T$, strong left thinness holds, and strong right thinness holds.

\section{PROOF}

A simple check shows that the first six examples used in the proof of Lemma 6 satisfy strong thinness. The seventh example violates right thinness and, hence, strong right thinness. It trivially satisfies strong left thinness. Similarly, the eighth example violates left thinness and, hence, strong left thinness. It trivially satisfies strong right thinness.

Using Lemma 5, we know that if a pair of disjoint relations $T$ and $F$, is such that $T$ is a semiorder, $R$ is a semiorder, TF $R^{s c} \subseteq T, R^{s c} F T \subseteq T$, and thinness holds, then the relation $\succsim_{\star}$ is complete. The following lemma takes note of some additional properties that obtain when thinness is replaced by strong thinness.

\section{Lemma 7}

Suppose that a pair of disjoint relations $T$ and $F$ is such that: $T$ is a semiorder, $R$ is a semiorder, $T F R^{s c} \subseteq T, R^{s c} F T \subseteq T$, and strong thinness holds. We have:

1. $x F y$ and $y \succ_{\star} z$ imply $x T z$,

2. $x \succ_{\star} y$ and $y F z$ imply $x T z$,

for all $x, y, z \in X$. 


\section{PROOF}

Part 1. By definition of $\succsim_{\star}$, we know that $x R z$. If $x F z$, strong right thinness and $x F y$ imply that $y \sim_{\star} z$, a contradiction. Part 2 is proved similarly using strong left thinness.

Hence, under the conditions of the above lemma, we have that, for all $x, y, z \in X$,

$$
\begin{aligned}
& x \succsim_{\star} y \text { and } y T z \Rightarrow x T z, \\
& x \succ_{\star} y \text { and } y F z \Rightarrow x T z, \\
& x \sim_{\star} y \text { and } y F z \Rightarrow x F z, \\
& x T y \text { and } y \succsim_{\star} z \Rightarrow x T z, \\
& x F y \text { and } y \succ_{\star} z \Rightarrow x T z, \\
& x F y \text { and } y \sim_{\star} z \Rightarrow x F z,
\end{aligned}
$$

This leads to our first result on representations with no nesting.

\section{Proposition 17}

Let $T$ and $F$ be a pair of disjoint relations on a finite or countably infinite set $X$. Let $R=T \cup F$. There are real-valued functions $u$ and $v$ on $X$ such that (36) holds iff $T$ is a semiorder, $R$ is a semiorder, TF $R^{s c} \subseteq T, R^{s c} F T \subseteq T$, and strong thinness holds. Furthermore the functions $u$ and $v$ can always be chosen so that, for all $x, y \in X$,

$$
x \succsim_{\star} y \Leftrightarrow u(x) \geq u(y) \Leftrightarrow v(x) \geq v(y) .
$$

\section{PROOF}

Necessity was noted earlier. Sufficiency is established as follows. Let us view the pair of relations $T$ and $F$ as relations between $X$ and $X^{\prime}$, a disjoint duplication of $X$. In view of (37), it is clear that (20) and (21) holds with $\succsim_{\star}$. Hence, we may apply the construction of Lemma 3. Because we have supposed that $X$ is countable, the weak order $\mathscr{L}$ has a numerical representation $F$. Defining $u$ and $v$ on $X$ as the restriction of $F$ on $X$ (resp. $X^{\prime}$ ) leads to a representation in which

$$
\begin{aligned}
x T y & \Leftrightarrow u(x)>v(y), \\
x F y & \Leftrightarrow u(x)=v(y), \\
x \succsim_{\star} y & \Leftrightarrow u(x) \geq u(y) \Leftrightarrow v(x) \geq v(y) .
\end{aligned}
$$

Since $R$ is irreflexive, we must have that $u(x)<v(x)$, which completes the proof.

We now turn to the general case. This will require order-denseness conditions that are inspired from the ones used in Nakamura (2002). 


\section{Proposition 18}

Let $T$ and $F$ be a pair of disjoint relations on a $X$. Define $R=T \cup F$. There are real-valued functions $u$ and $v$ on $X$ such that (36) holds iff $T$ is a semiorder, $R$ is a semiorder, $T F R^{s c} \subseteq T, R^{s c} F T \subseteq T$, strong thinness holds, and there is a finite or countably infinite subset $\mathcal{X}^{*} \subseteq X$ such that, for all $x, y \in X$,

$$
\begin{aligned}
x T y & \Rightarrow\left[x \succsim_{\star} x^{*}, x^{*} T y^{*}, y^{*} \succsim_{\star} y\right], \\
x R^{c} y & \Rightarrow\left[x^{*} \succsim_{\star} x, x^{*} R^{c} y^{*}, y \succsim_{\star} y^{*}\right],
\end{aligned}
$$

for some $x^{*}, y^{*} \in \mathcal{X}^{*}$. Furthermore the functions $u$ and $v$ can always be chosen so that (38) holds.

\section{PROOF}

Necessity. The necessity of the first six conditions is easily shown. Let us prove that model (36) implies (39) and (40).

Let $\lambda_{j} \in u(X)$ be such that

$$
\mu_{j}<\lambda_{j} \text { and }\left(\mu_{j}, \lambda_{j}\right) \cap u(X)=\varnothing,
$$

for some $\mu_{j} \in v(X)$. With each such $\lambda_{j} \in u(X)$, we associate a particular $\mu_{j} \in$ $v(X)$ such that (41) holds. Suppose that $\lambda_{k}, \lambda_{j} \in u(X)$ both satisfy (41) and $\lambda_{k}<\lambda_{j}$. The two intervals $\left(\mu_{k}, \lambda_{k}\right)$ and $\left(\mu_{j}, \lambda_{j}\right)$ are disjoint since $\mu_{j}<\lambda_{k}$ would violate the fact that $\left(\mu_{j}, \lambda_{j}\right) \cap u(X)=\varnothing$. The collection of the numbers $\lambda_{j}$ must be countable because the intervals $\left(\mu_{j}, \lambda_{j}\right)$ are nonempty and disjoint and, therefore, each one contains a distinct rational number. Therefore, there is a finite or countably infinite set $\mathcal{X}_{1}^{*} \subseteq X$ such that $u\left(\mathcal{X}_{1}^{*}\right)$ contains all the $\lambda_{j}$.

Let $\lambda_{j} \in u(X)$ be such that

$$
\lambda_{j}<\mu_{j} \text { and }\left(\lambda_{j}, \mu_{j}\right) \cap u(X)=\varnothing,
$$

for some $\mu_{j} \in v(X)$. With each such $\lambda_{j} \in u(X)$, we associate a particular $\mu_{j} \in$ $v(X)$ such that (42) holds. Suppose that $\lambda_{k}, \lambda_{j} \in u(X)$ both satisfy (42) and $\lambda_{k}<\lambda_{j}$. The two intervals $\left(\lambda_{k}, \mu_{k}\right)$ and $\left(\lambda_{j}, \mu_{j}\right)$ are disjoint since $\lambda_{j}<\mu_{k}$ would violate the fact that $\left(\lambda_{k}, \mu_{k}\right) \cap u(X)=\varnothing$. The collection of the numbers $\lambda_{j}$ must be countable because the intervals $\left(\lambda_{j}, \mu_{j}\right)$ are nonempty and disjoint and, therefore, each one contains a distinct rational number. Therefore, there is a finite or countably infinite set $\mathcal{X}_{2}^{*} \subseteq X$ such that $u\left(\mathcal{X}_{2}^{*}\right)$ contains all the $\lambda_{j}$.

Let $\mu_{j} \in v(X)$ be such that

$$
\mu_{j}<\lambda_{j} \text { and }\left(\mu_{j}, \lambda_{j}\right) \cap v(X)=\varnothing,
$$

for some $\lambda_{j} \in u(X)$. With each such $\mu_{j} \in v(X)$, we associate a particular $\lambda_{j} \in$ $u(X)$ such that (43) holds. Suppose that $\mu_{j}, \mu_{k} \in v(X)$ both satisfy (43) and 
$\mu_{j}<\mu_{k}$. The two intervals $\left(\mu_{j}, \lambda_{j}\right)$ and $\left(\mu_{k}, \lambda_{k}\right)$ are disjoint since $\mu_{k}<\lambda_{j}$ would violate the fact that $\left(\mu_{j}, \lambda_{j}\right) \cap v(X)=\varnothing$. The collection of the numbers $\mu_{j}$ must be countable because the intervals $\left(\mu_{j}, \lambda_{j}\right)$ are nonempty and disjoint and, therefore, each one contains a distinct rational number. Therefore, there is a finite or countably infinite set $\mathcal{X}_{3}^{*} \subseteq X$ such that $v\left(\mathcal{X}_{3}^{*}\right)$ contains all the $\mu_{j}$.

Let $\mu_{j} \in v(X)$ be such that

$$
\lambda_{j}<\mu_{j} \text { and }\left(\lambda_{j}, \mu_{j}\right) \cap v(X)=\varnothing,
$$

for some $\lambda_{j} \in u(X)$. With each such $\mu_{j} \in v(X)$, we associate a particular $\lambda_{j} \in$ $u(X)$ such that (44) holds. Suppose that $\mu_{j}, \mu_{k} \in v(X)$ both satisfy (44) and $\mu_{j}<\mu_{k}$. The two intervals $\left(\lambda_{j}, \mu_{j}\right)$ and $\left(\lambda_{k}, \mu_{k}\right)$ are disjoint since $\mu_{j}>\lambda_{k}$ would violate the fact that $\left(\lambda_{k}, \mu_{k}\right) \cap v(X)=\varnothing$. The collection of the numbers $\mu_{j}$ must be countable because the intervals $\left(\lambda_{j}, \mu_{j}\right)$ are nonempty and disjoint and, therefore, each one contains a distinct rational number. Therefore, there is a finite or countably infinite set $\mathcal{X}_{4}^{*} \subseteq X$ such that $v\left(\mathcal{X}_{4}^{*}\right)$ contains all the $\mu_{j}$.

Let us select a subset $\mathcal{X}_{5}^{*} \subseteq X$ such that for every pair of rational numbers $p$ and $q$ such that $p<q$ the following condition holds:

$$
(p, q) \cap u(X) \neq \varnothing \Rightarrow\left[p<u\left(x^{*}\right)<q \text {, for some } x^{*} \in \mathcal{X}_{5}^{*}\right] .
$$

It is easy to see that the set $\mathcal{X}_{5}^{*} \subseteq X$ can always be taken to be finite or countably infinite.

Let us finally select a subset $\mathcal{X}_{6}^{*} \subseteq X$ such that for every pair of rational numbers $p$ and $q$ such that $p<q$ the following condition holds:

$$
(p, q) \cap v(X) \neq \varnothing \Rightarrow\left[p<v\left(x^{*}\right)<q \text {, for some } x^{*} \in \mathcal{X}_{6}^{*}\right] .
$$

It is easy to see that the set $\mathcal{X}_{6}^{*} \subseteq X$ can always be taken to be finite or countably infinite.

Define $\mathcal{X}^{*}=\mathcal{X}_{1}^{*} \cup \mathcal{X}_{2}^{*} \cup \mathcal{X}_{3}^{*} \cup \mathcal{X}_{4}^{*} \cup \mathcal{X}_{5}^{*} \cup \mathcal{X}_{6}^{*}$. By construction, $\mathcal{X}^{*} \subseteq X$ is finite or countably infinite. Let us show that $\mathcal{X}^{*}$ satisfies (39) and (40).

Let us first show that (39) holds. Suppose that $x T y$, so that $u(x)>v(y)$. We distinguish two cases.

1. If $(v(y), u(x)) \cap u(X)=\varnothing$ then, by construction, we have $u(x)=u\left(x^{*}\right)$, for some $x^{*} \in \mathcal{X}_{1}^{*}$. Because $u(x)=u\left(x^{*}\right)>v(y)$, we clearly have $x \succsim_{\star} x^{*}$ and $x^{*} T y$.

2. Otherwise we have $(v(y), u(x)) \cap u(X) \neq \varnothing$ and let $z$ be any element in $X$ such that $v(y)<u(z)<u(x)$. Let $p, q \in \mathbb{Q}$ be such that $v(y)<p<u(z)<q<$ $u(x)$. By construction of the set $\mathcal{X}_{5}^{*}$, we have $v(y)<p<u\left(x^{*}\right)<q<u(x)$, for some $x^{*} \in \mathcal{X}_{5}^{*}$. Because $u(x)>u\left(x^{*}\right)>v(y)$, we have $x \succsim_{\star} x^{*}$ and $x^{*} T y$. 
In either case we have $x \succsim x^{*}, x^{*} T y$, and $u\left(x^{*}\right)>v(y)$. Again, we distinguish two cases.

1. If $\left(v(y), u\left(x^{*}\right)\right) \cap v(X)=\varnothing$ then, by construction, we have $v(y)=v\left(y^{*}\right)$, for some $y^{*} \in \mathcal{X}_{3}^{*}$. Because $v(y)=v\left(y^{*}\right)<v\left(x^{*}\right)$, we have $y^{*} \succsim_{\star} y$ and $x^{*} T y^{*}$. Hence we have $x \succsim_{\star} x^{*}, x^{*} T y^{*}$, and $y^{*} \succsim_{\star} y$, as required.

2. Otherwise, we have $\left(v(y), u\left(x^{*}\right)\right) \cap v(X) \neq \varnothing$ and let $z$ be any element in $X$ such that $v(y)<v(z)<u\left(x^{*}\right)$. Let $p, q \in \mathbb{Q}$ be such that $v(y)<p<v(z)<$ $q<u\left(x^{*}\right)$. By construction of the set $\mathcal{X}_{6}^{*}$, we have $v(y)<p<v\left(y^{*}\right)<q<$ $u\left(x^{*}\right)$, for some $y^{*} \in \mathcal{X}_{6}^{*}$. Because $u\left(x^{*}\right)>v\left(y^{*}\right)>v(y)$, we have $x^{*} T y^{*}$ and $y^{*} \succsim_{\star} y$. Hence we have $x \succsim_{\star} x^{*}, x^{*} T y^{*}$, and $y^{*} \succsim_{\star} y$, as required.

Let us now show that (40) holds. Suppose now that $x R^{c} y$, so that $u(x)<v(y)$. We distinguish two cases.

1. If $(u(x), v(y)) \cap v(X)=\varnothing$ then, by construction, we have $v(y)=v\left(y^{*}\right)$, for some $y^{*} \in \mathcal{X}_{4}^{*}$. Because $v(y)=v\left(y^{*}\right)>u(x)$, we clearly have $y \succsim_{\star} y^{*}$ and $x R^{c} y^{*}$.

2. Otherwise we have $(u(x), v(y)) \cap v(X) \neq \varnothing$ and let $z$ be any element in $X$ such that $u(x)<v(z)<v(y)$. Let $p, q \in \mathbb{Q}$ be such that $u(x)<p<v(z)<q<$ $v(y)$. By construction of the set $\mathcal{X}_{6}^{*}$, we have $u(x)<p<v\left(y^{*}\right)<q<v(y)$, for some $y^{*} \in \mathcal{X}_{6}^{*}$. Because $u(x)<v\left(y^{*}\right)<v(y)$, we have $y \succsim_{\star} y^{*}$ and $x R^{c} y^{*}$.

In either case we have $y \succsim_{\star} y^{*}, x R^{c} y^{*}$, and $u(x)<v\left(y^{*}\right)$. Again, we distinguish two cases.

1. If $\left(u(x), v\left(y^{*}\right)\right) \cap u(X)=\varnothing$ then, by construction, we have $u(x)=u\left(x^{*}\right)$, for some $x^{*} \in \mathcal{X}_{2}^{*}$. Because $u(x)=u\left(x^{*}\right)<v\left(y^{*}\right)$, we have $x^{*} \succsim_{\star} x$ and $x^{*} R^{c} y^{*}$. Hence we have $x^{*} \succsim_{\star} x, x^{*} R^{c} y^{*}$, and $y \succsim_{\star} y^{*}$, as required.

2. Otherwise, we have $\left(u(x), v\left(y^{*}\right)\right) \cap u(X) \neq \varnothing$ and let $z$ be any element in $X$ such that $u(x)<u(z)<v\left(y^{*}\right)$. Let $p, q \in \mathbb{Q}$ be such that $u(x)<p<u(z)<$ $q<v\left(y^{*}\right)$. By construction of the set $\mathcal{X}_{5}^{*}$, we have $u(x)<p<u\left(x^{*}\right)<q<$ $v\left(y^{*}\right)$, for some $x^{*} \in \mathcal{X}_{5}^{*}$. Because $u(x)<u\left(x^{*}\right)<v\left(y^{*}\right)$, we have $x^{*} \succsim_{\star} x$ and $x^{*} R^{c} y^{*}$. Hence we have $x^{*} \succsim_{\star} x, x^{*} R^{c} y^{*}$, and $y \succsim_{\star} y^{*}$, as required.

Sufficiency. Let us view the pair of relations $T$ and $F$ as relations between $X$ and $X^{\prime}$, a disjoint duplication of $X$. In view of (37), it is clear that ${ }_{\star}$ on $X$ satisfies (20) and that $\succsim_{\star}$ on $X^{\prime}$ satisfies (21). Hence, we may apply the construction of Lemma 3 showing that the relation $\mathscr{L}$ on $X \cup X^{\prime}$ is a weak order. In what follows, 
we consider the weak order $\mathscr{L}$ with $\succsim_{b}^{\ell}=\succsim_{\star}$ and $\succsim_{b}^{r}=\succsim_{\star}$. The proof will be complete if we show that there is a countable subset $\mathcal{Y}^{*}$ of $X \cup X^{\prime}$ that is dense for $\mathscr{L}$, i.e., that, for all $\alpha, \beta \in X \cup X^{\prime}$,

$$
\alpha \mathscr{L}^{a} \beta \Rightarrow\left[\alpha \mathscr{L} \gamma \text { and } \gamma \mathscr{L} \beta, \text { for some } \gamma \in \mathcal{Y}^{*}\right]
$$

There are four cases to consider.

1. Suppose that $\alpha, \beta \in X$. Then $\alpha \mathscr{L}^{a} \beta$ means that $\alpha \succ_{\star} \beta$. This implies either $\alpha \succ_{T}^{\ell} \beta$ or $\alpha \succ_{R}^{r} \beta$.

Suppose first that $\alpha \succ_{T}^{\ell} \beta$. This implies that we have $\alpha T \gamma$ and $\operatorname{Not}[\beta T \gamma]$, for some $\gamma \in X^{\prime}$. We know that $\alpha T \gamma$ implies that $\alpha \succsim_{\star} \alpha^{*}, \alpha^{*} T \gamma^{*}, \gamma^{*} \succsim_{\star} \gamma$, for some $\alpha^{*} \in \mathcal{X}^{*} \cap X$ and some $\gamma^{*} \in \mathcal{X}^{*} \cap X^{\prime}$. Because $\alpha^{*} T \gamma^{*}$ and $\gamma^{*} \succsim_{\star} \gamma$, we know that $\alpha^{*} T \gamma$. Since $\operatorname{Not}[\beta T \gamma]$, we have $\alpha^{*} \succ_{\star} \beta$. Hence we have $\alpha \succsim_{\star} \alpha^{*}$ and $\alpha^{*} \succ_{\star} \beta$, for some $\alpha^{*} \in \mathcal{X}^{*} \cap X$. This implies $\alpha \mathscr{L} \alpha^{*}$ and $\alpha^{*} \mathscr{L} \beta$.

Suppose now that $\alpha \succ_{R}^{\ell} \beta$. This implies that we have $\alpha R \gamma$ and $\operatorname{Not}[\beta R \gamma]$, for some $\gamma \in X^{\prime}$. We know that $\beta R^{c} \gamma$ implies that $\beta^{*} \succsim_{\star} \beta, \beta^{*} R^{c} \gamma^{*}$, $\gamma \succsim \star \gamma^{*}$, for some $\beta^{*} \in \mathcal{X}^{*} \cap X$ and some $\gamma^{*} \in \mathcal{X}^{*} \cap X^{\prime}$. Because $\alpha R \gamma$ and $\gamma{ }_{\star} \gamma^{*}$, we know that $\alpha R \gamma^{*}$. Because we know that $\beta^{*} R^{c} \gamma^{*}$, we must have $\alpha \succ_{\star} \beta^{*}$. Hence we have $\alpha \succsim_{\star} \beta^{*}$ and $\beta^{*} \succsim_{\star} \beta$, for some $\beta^{*} \in \mathcal{X}^{*} \cap X$. This implies $\alpha \mathscr{L} \beta^{*}$ and $\beta^{*} \mathscr{L} \beta$.

2. Suppose that $\alpha, \beta \in X^{\prime}$. The proof is clearly similar to the preceding case.

3. Suppose that $\alpha \in X$ and $\beta \in X^{\prime}$. Then $\alpha \mathscr{L}^{a} \beta$ means that $\alpha T \beta$. We know that $\alpha T \beta$ implies that $\alpha \succsim_{\star} \alpha^{*}, \alpha^{*} T \beta^{*}, \beta^{*} \succsim_{\star} \beta$, for some $\alpha^{*} \in \mathcal{X}^{*} \cap X$ and some $\beta^{*} \in \mathcal{X}^{*} \cap X^{\prime}$. Since $\alpha^{*} T \beta^{*}$ and $\beta^{*} \succsim_{\star} \beta$, we obtain $\alpha^{*} T \beta$. Hence, we have $\alpha \succsim_{\star} \alpha^{*}$ and $\alpha^{*} T \beta$, for some $\alpha^{*} \in \mathcal{X}^{*} \cap X$. This implies $\alpha \mathscr{L} \alpha^{*}$ and $\alpha^{*} \mathscr{L} \beta$.

4. Suppose that $\alpha \in X^{\prime}$ and $\beta \in X$. Then $\alpha \mathscr{L}^{a} \beta$ means that $\beta R^{c} \alpha$. We know that $\beta R^{c} \alpha$ implies that $\beta^{*} \succsim_{\star} \beta, \beta^{*} R^{c} \alpha^{*}, \alpha \succsim_{\star} \alpha^{*}$, for some $\beta^{*} \in \mathcal{X}^{*} \cap X$ and some $\alpha^{*} \in \mathcal{X}^{*} \cap X^{\prime}$. Since $\beta^{*} \succsim_{\star} \beta$ and $\beta^{*} R^{c} \alpha^{*}$, we have that $\beta R^{c} \alpha^{*}$. Hence we have $\alpha \succsim_{\star} \alpha^{*}$ and $\beta R^{c} \alpha^{*}$, for some $\alpha^{*} \in \mathcal{X}^{*} \cap X^{\prime}$. This implies $\alpha \mathscr{L} \alpha^{*}$ and $\alpha^{*} \mathscr{L} \beta$. 


\subsection{Constant threshold representations}

In a constant threshold representation, there is a real-valued function $u$ on $X$ such that, for all $x, y \in X$,

$$
\begin{aligned}
& x T y \Leftrightarrow u(x)>u(y)+1, \\
& x F y \Leftrightarrow u(x)=u(y)+1 .
\end{aligned}
$$

It will first be useful to note additional implications of the conditions introduced above.

\section{Lemma 8}

Let $T$ and $F$ be a pair of disjoint relations on a set $X$. If $T$ is a semiorder, $R$ is a semiorder, $T F R^{s c} \subseteq T, R^{s c} F T \subseteq T$, strong thinness holds, then we have:

$$
F F \subseteq T, \quad F T \subseteq T, \quad T F \subseteq T .
$$

Furthermore, we have:

$$
\begin{aligned}
& R^{s c} T T \subseteq T \quad T R^{s c} T \subseteq T \quad T T R^{s c} \subseteq T, \\
& F^{d} T T \subseteq T \quad T F^{d} T \subseteq T \quad T T F^{d} \subseteq T, \\
& R^{s c} T F \subseteq T \quad F T R^{s c} \subseteq T \quad F R^{s c} T \subseteq T \quad T R^{s c} F \subseteq T, \\
& F^{d} F T \subseteq T \quad F^{d} T F \subseteq T \quad F F^{d} T \subseteq T \quad F T F^{d} \subseteq T \quad T F F^{d} \subseteq T \quad T F^{d} F \subseteq T, \\
& R^{s c} F F \subseteq T \quad F R^{s c} F \subseteq T \quad F F R^{s c} \subseteq T, \\
& F^{d} F F \subseteq F \quad F F^{d} F \subseteq F \quad F F F^{d} \subseteq F .
\end{aligned}
$$

\section{ProOF}

Let us show that $F F \subseteq T$. Suppose that $a F b$ and $b F c$. Since $R$ is a semiorder, we know that $a R c$. Suppose that $a F c$. Since $b F c$, strong left thinness implies that $a \sim_{\star} b$. Since $a F b$, this implies $a F a$, contradicting the irreflexivity of $F$. The proof that $F T \subseteq T$ and $T F \subseteq T$ is similar.

That $R^{s c} T T \subseteq T, T R^{s c} T \subseteq T, T T R^{s c} \subseteq T, F^{d} T T \subseteq T, T F^{d} T \subseteq T, T T F^{d} \subseteq$ $T$ are immediate consequences of the fact that $T$ is a semiorder.

By hypothesis, we know that $R^{s c} F T \subseteq T$ and $T F R^{s c} \subseteq T$. Lemma 5 has shown that $F R^{s c} T \subseteq T$ and $T R^{s c} F \subseteq T$. Let us show that $R^{s c} F T \subseteq T$. Because $R$ is a semiorder, $a R^{s c} b, b F c$ and $c T d$ imply that $a R d$. If $a F d, c F d$ and strong left thinness imply $a \sim_{\star} c$. This contradicts the fact that $b R^{s c} a$ and $b F c$. The proof that $R^{s c} T F \subseteq T$ is similar.

Let us show that $F^{d} F T \subseteq T$. Suppose that $a F^{d} b, b F c$, and $c T d$. Hence, we have $b F a$ and $b F c$, so that strong right thinness implies $a \sim_{\star} c$. Hence $c T d$ implies $a T d$. The proofs that $F^{d} T F \subseteq T, F F^{d} T \subseteq T, F T F^{d} \subseteq T, T F F^{d} \subseteq T$, and $T F^{d} F \subseteq T$ are similar.

Let us show that $R^{s c} F F \subseteq T$. Suppose that $a R^{s c} b, b F c$, and $c F d$. Because $R$ is a semiorder, we know that $a R d$. Suppose that $a F d$. Using $c F d$ and 
strong left thinness, we obtain $a \sim_{\star} c$, so that $b F c$ implies $b F a$, a contradiction. The proofs that $F R^{s c} F \subseteq T$ and $F F R^{s c} \subseteq T$ are similar.

Let us finally prove that $F^{d} F F \subseteq F$. Suppose that $a F^{d} b, b F c$, and $c F d$. Hence, we have $b F a$ and $b F c$, so that strong right thinness implies $a \sim_{\star} c$. Hence $c F d$ implies $a F d$, as required. The proofs that $F F^{d} F \subseteq F$ and $F F F^{d} \subseteq F$ are similar.

This leads to our main result on representations with constant threshold.

\section{Proposition 19}

Let $T$ and $F$ be a pair of disjoint relations on a finite set $X$. Let $R=T \cup F$. There is a real-valued function $u$ on $X$ such that (47) holds iff $T$ is a semiorder, $R$ is a semiorder, $T F R^{s c} \subseteq T, R^{s c} F T \subseteq T$, and strong thinness holds. Furthermore the function $u$ can always be chosen so that, for all $x, y \in X$,

$$
x \succsim_{\star} y \Leftrightarrow u(x) \geq u(y) .
$$

\section{ProOF}

Necessity results from Proposition 17. We show sufficiency. We use the following result from Graph Theory (see Roy, 1970, Theorem VIII.1, page 270 or Schrijver, 2003, page 108). Let $G=(A, U)$ be a finite digraph, $A$ being the set of nodes and $U \subseteq A^{2}$ being the set of arcs. If $(a, b) \in U$, we say that $a$ (resp. $b$ ) is the initial (resp. terminal) extremity of the arc $(a, b)$. Let $\sigma$ be a real-valued function on $U$. There is a real-valued function $f$ on $A$ such that, for all $a, b \in A$,

$$
f(a)-f(b) \geq \sigma(a, b)
$$

iff this valued digraph has no circuit of strictly positive value, the value of a circuit being the sum of the values $\sigma(a, b)$ on the arcs $(a, b)$ belonging to the circuit. It is clear that, in the above result, we may restrict our attention to elementary circuits, i.e., each node visited by the circuit is the initial extremity of exactly one arc in the circuit and the terminal extremity of exactly one arc in the circuit.

Given a pair of relations $T$ and $F$ on $X$, we build a digraph having $X$ for set of nodes. Let $\varepsilon \in \mathbb{R}$. The set of arcs and the function $\sigma$ are as follows. If $x T y$, we add an $\operatorname{arc}(x, y)$ with value $1+\varepsilon$. If $x F y$, we add an $\operatorname{arc}(x, y)$ with value 1 and an $\operatorname{arc}(y, x)$ with value -1 . If $x R^{s c} y$, we add an $\operatorname{arc}(x, y)$ with value $-1+\varepsilon$ and an $\operatorname{arc}(y, x)$ with value $-1+\varepsilon$.

The length of a circuit is the number of its arcs. Let $\bar{n}$ be the the length of an elementary circuit of maximum length in this digraph. We take $\varepsilon$ such that $0<\varepsilon<1 / \bar{n}$. 
Any function $f$ on this digraph satisfying (49) will be such that, for all $x, y \in X$,

$$
\begin{aligned}
x T y & \Rightarrow f(x)-f(y) \geq 1+\varepsilon \Rightarrow f(x)-f(y)>1, \\
x F y & \Rightarrow\left\{\begin{array}{l}
f(x)-f(y) \geq 1 \\
f(y)-f(x) \geq 1
\end{array}\right\} \Rightarrow f(x)-f(y)=1, \\
x R^{s c} y & \Rightarrow\left\{\begin{array}{l}
f(x)-f(y) \geq-1+\varepsilon \\
f(y)-f(x) \geq-1+\varepsilon
\end{array}\right\} \Rightarrow|f(x)-f(y)|<1 .
\end{aligned}
$$

Since for each ordered pair $(x, y) \in X^{2}$, we have one and only one of: $x T y, x F y$, $x R^{s c} y, x F^{d} y$ and $x T^{d} y$, we obtain a numerical representation of $T$ and $F$ in model (47) with $u=f$.

The value of a circuit of length $n=n_{T}+n_{F}+n_{F^{d}}+n_{R^{s c}}$ in this valued digraph is given by

$$
\left(n_{T}+n_{F}-n_{F^{d}}-n_{R^{s c}}\right)+\varepsilon\left(n_{T}+n_{R^{s c}}\right)
$$

where $n_{T}$ (resp. $n_{F}, n_{F^{d}}, n_{R^{s c}}$ ) is the number of arcs of type $T$ (resp. $F, F^{d}, R^{s c}$ ). We show below that the digraph cannot contain an elementary circuit having a strictly positive value.

Let us first observe that a circuit in this digraph containing only arcs of type $T$ or of type $F$ would contradict the irreflexivity of $R$. Hence, the digraph cannot contain a circuit of length 1 (i.e., a loop) having strictly positive value. It is simple to check that the digraph cannot contain a circuit of length 2 having a strictly positive value (this easily results from the disjointness of $T$ and $F$, the definition of $R^{s c}$, and the fact that $R$ is a semiorder).

Suppose that the digraph contains a circuit of length $n \geq 3$ having a strictly positive value. We prove that this is contradictory. We distinguish two cases.

Case 1. Suppose that $n_{T}+n_{R^{s c}}=0$. Because the value of the circuit is strictly positive, we must have $n_{F}>n_{F^{d}}$. Since we know that the circuit cannot contain only arcs of type $F$, we have at least one configuration of type $F F^{d}$ or of type $F^{d} F$. Because $F F^{d} \subseteq \sim_{\star}$ and $F^{d} F \subseteq \sim_{\star}$, in either case, we can build a circuit of length $n-2$ (e.g., the three $\operatorname{arcs} a F b, b F^{d} c$ and $c F d$ are replaced by an arc $a F d$ in the new circuit). This shorter circuit (of length $n-2$ ) only contains arcs of type $F$ and of type $F^{d}$. Compared to the initial circuit, we have reduced by one both the number of arcs of type $F$ and the number of arcs of type $F^{d}$. Repeating the process, we keep eliminating the arcs of type $F^{d}$. At some step, we will obtain a circuit only containing arcs of type $F$, which is impossible.

Case 2. Suppose that $n_{T}+n_{R^{s c}}>0$. Since the circuit has a strictly positive value, we must have $n_{T}+n_{F} \geq n_{F^{d}}+n_{R^{s c}}$ (if $n_{T}+n_{F}<n_{F^{d}}+n_{R^{s c}}$, supposing that $\left(n_{T}+n_{F}-n_{F^{d}}-n_{R^{s c}}\right)+\varepsilon\left(n_{T}+n_{R^{s c}}\right)>0$ implies $\varepsilon>1 /\left(n_{T}+n_{R^{s c}}\right)$, which 
is impossible since $\varepsilon<1 / \bar{n})$. Hence, the circuit contains at least as many arcs of type $T$ or $F$ as arcs of type $F^{d}$ or $R^{s c}$. Because we cannot have only arcs of type $T$ or of type $F$, there is at least one arc of type $F^{d}$ or $R^{s c}$. We distinguish two cases.

a) Suppose first that the circuit contains at least one arc of type $T$, i.e., that $n_{T}>0$. Because $n_{T}+n_{F} \geq n_{F^{d}}+n_{R^{s c}}$, the circuit contains a sequence of one of the following three types: $\alpha \beta \beta, \beta \alpha \beta$, or $\beta \beta \alpha$ with $\alpha$ being either $R^{s c}$ or $F^{d}$ and $\beta$ being either $T$ or $F$. Using Lemma 8 , we can build a circuit of length $n-2$ still containing at least as many arcs of type $F$ or $T$ as arcs of type $R^{s c}$ or $F^{d}$. If, in the sequence, at least one of the two $\beta$ is $T$, we know that the arc that replaces the three suppressed ones is of type $T$. Hence, since we still have $n_{T}>0$ in the new shorter circuit. If the sequence $\alpha \beta \beta, \beta \alpha \beta$, or $\beta \beta \alpha$ is of the type $R^{s c} F F, F R^{s c} F$, or $F F R^{s c}$ the arc that replaces the three suppressed ones is of type $T$, so that we still have $n_{T}>0$ in the new shorter circuit. If the sequence $\alpha \beta \beta, \beta \alpha \beta$, or $\beta \beta \alpha$ is of the type $F^{d} F F, F F^{d} F$, or $F F F^{d}$ the added arc is of the type $F$. Hence the number of $\operatorname{arcs}$ of type $T$ and $R^{s c}$ in the new circuit remain unchanged and $n_{T}>0$ still holds in the new shorter circuit. We can therefore repeat the above process. Doing so, we build a sequence of circuits in which $n_{F^{d}}+n_{R^{s c}}$ is decreased by one unit at each step. If, at some step, $n_{F^{d}}+n_{R^{s c}}$ becomes 0 , we have built a circuit containing only arcs of type $T$ and $F$, which is impossible. Otherwise, we will obtain a circuit of length 1 or 2 that has a strictly positive value, which is also impossible.

b) Suppose now that the circuit does not contain arcs of type $T$, which implies that there is at least one arc of type $R^{s c}$, i.e., $n_{R^{s c}}>0$. Because there at least as many arcs of type $F$ than of arcs of type $F^{d}$ or $R^{s c}$, the circuit contains at least one configuration of the type $\alpha \beta \beta, \beta \alpha \beta$, or $\beta \beta \alpha$ with $\alpha$ being either $R^{s c}$ or $F^{d}$ and $\beta$ being $F$. We distinguish two cases.

(i) If $\alpha$ is $R^{s c}$, we know, using Lemma 8, that $R^{s c} F F \subseteq T, F R^{s c} F \subseteq T$ and $F F R^{s c} \subseteq T$. We can therefore build a circuit of length $n-2$ such that $n_{T}+n_{F} \geq n_{F^{d}}+n_{R^{s c}}$ and $n_{T}>0$ (e.g., the three $\operatorname{arcs} a R^{s c} b, b F c$, and $c F d$ are replaced by an arc $a T d)$. Hence, we are back to case a).

(ii) If $\alpha$ is $F^{d}$, we know, using Lemma 8, that $F^{d} F F \subseteq F, F F^{d} F \subseteq F$ and $F F F^{d} \subseteq F$. We can therefore build a circuit of length $n-2$ in which we have reduced by one the number of arcs of type $F^{d}$ and the number of arcs of type $F$. Repeating the process, we keep eliminating arcs of type $F^{d}$. Because the initial circuit contains at least one arc of type $R^{s c}$, we will be confronted, at some step, with a configuration of the type $\alpha \beta \beta$, $\beta \alpha \beta$, or $\beta \beta \alpha$ with $\alpha$ being $R^{s c}$ and $\beta$ being $F$. Using Lemma 8 , we now 
that $R^{s c} F F \subseteq T, F R^{s c} F \subseteq T$ and $F F R^{s c} \subseteq T$. We can therefore build a shorter circuit replacing the three arcs in the sequence by an arc of type $T$. In this shorter circuit, we have decreased the number of $F$ arcs by 2 , decreased the number of $R^{s c}$ arcs by 1 and added one arc of type $T$. In this new circuit, we therefore have $n_{T}>0$ and $n_{T}+n_{F} \geq n_{F^{d}}+n_{R^{s c}}$ and we are back to case a).

Hence the digraph cannot contain a circuit of strictly positive value, which proves the existence of a representation of type (47).

To complete the proof, observe that the above reasoning can be applied to the set $X / \sim_{\star}$ of equivalence classes of $X$ under $\sim_{\star}$. Doing so, leads to build a numerical representation in model (47) in which $u$ is a numerical representation of the weak order ${ }_{\star}$.

Combining Propositions 17 and 19, shows that on finite sets, representations with no nesting and constant threshold representations are equivalent, as was the case when there is no frontier.

We have not investigated the generalization of constant threshold representation to countable and uncountable sets, which could be the subject of future research.

\section{Applications: conjoint measurement and tem- poral logic}

Our initial motivation for studying biorders with frontier was the following. Let $X=X_{1} \times X_{2} \times \cdots \times X_{n}$ be a set of objects evaluated on $n$ attributes. Traditional conjoint measurement (Krantz, Luce, Suppes, and Tversky, 1971, Ch. 6) starts with a binary relation $\succsim$ on $X$ and looks for an additive numerical representation of this relation, i.e., for real-valued functions $u_{i}$ on $X_{i}$ such that, for all $x, y \in X$,

$$
x \succsim y \Leftrightarrow \sum_{i=1}^{n} u_{i}\left(x_{i}\right) \geq \sum_{i=1}^{n} u_{i}\left(y_{i}\right),
$$

where $x=\left(x_{1}, x_{2}, \ldots, x_{n}\right)$ and $y=\left(y_{1}, y_{2}, \ldots, y_{n}\right)$

In Bouyssou and Marchant $(2009,2010)$ we study measurement models in which the binary relation $\succsim$ is replaced with an ordered partition or an ordered covering $\left\langle C^{1}, C^{2}, \ldots, C^{r}\right\rangle$ of the set of objects (a similar problem was first studied in Goldstein, 1991. This analysis was pursued in Słowiński, Greco, and Matarazzo, 2002 and Bouyssou and Marchant, 2007a,b). In such a setting, we know that objects belonging to $C^{k+1}$ are better than objects belonging to $C^{k}$ but we have 
no information on the way two objects belonging to the same category compare in terms of preference. This amounts to working with a much poorer information than in the classic setting with a complete binary relation $\succsim$.

Consider first an ordered partition $\left\langle C^{1}, C^{2}, \ldots, C^{r}\right\rangle$ (i.e., the sets $C^{k}$ are nonempty, pairwise disjoint and their union is the entire set $X)$. In this case, we are interested in finding real-valued functions $u_{i}$ on $X_{i}$ such that, for all $x \in X$ and all $k \in\{1,2, \ldots, r\}$,

$$
x \in C^{k} \Leftrightarrow \sigma^{k-1} \leq \sum_{i=1}^{n} u_{i}\left(x_{i}\right)<\sigma^{k},
$$

with the convention that $\sigma^{0}=-\infty, \sigma^{r}=+\infty$ and where $\sigma^{1}, \sigma^{2}, \ldots, \sigma^{r-1}$ are real numbers such that $\sigma^{1}<\sigma^{2}<\cdots<\sigma^{r-1}$.

When there are only two attributes and two categories, the above problem is highly degenerate and our problem relates more to ordinal than to conjoint measurement. Indeed, in such a case, the problem clearly reduces to finding realvalued functions $u_{1}$ on $X_{1}$ and $u_{2}$ on $X_{2}$ such that, for all $x \in X$,

$$
x \in C^{2} \Leftrightarrow u_{1}\left(x_{1}\right)+u_{2}\left(x_{2}\right)>0 .
$$

Define the relation $\mathcal{T}$ between the sets $X_{1}$ and $X_{2}$ letting, for all $x_{1} \in X_{1}$ and all $x_{2} \in X_{2}$,

$$
x_{1} \mathcal{T} x_{2} \Leftrightarrow\left(x_{1}, x_{2}\right) \in C^{2}
$$

It is clear that asking for a representation in model (50) is equivalent to asking for the existence of two functions $f$ on $X_{1}$ and $g$ on $X_{2}$ such that

$$
x_{1} \mathcal{T} x_{2} \Leftrightarrow f\left(x_{1}\right)>g\left(x_{2}\right),
$$

and Proposition 2 gives necessary and sufficient conditions for that.

In model (50), all objects belong either to $C^{2}$ or to $C^{1}$. A simple extension consists in removing the condition that $C^{2} \cap C^{1}=\varnothing$, therefore allowing for an hesitation between two consecutive categories. Let $C_{>}^{2}=C^{2} \backslash C^{1}$. With such a model, we are looking for a numerical representation such that, for all $x \in X$,

$$
\begin{aligned}
x \in C_{>}^{2} & \Leftrightarrow u_{1}\left(x_{1}\right)+u_{2}\left(x_{2}\right)>0, \\
x \in C^{2} \cap C^{1} & \Leftrightarrow u_{1}\left(x_{1}\right)+u_{2}\left(x_{2}\right)=0 .
\end{aligned}
$$

Define the relations $\mathcal{T}$ and $\mathcal{F}$ between the sets $X_{1}$ and $X_{2}$ letting, for all $x_{1} \in X_{1}$ and all $x_{2} \in X_{2}$,

$$
\begin{aligned}
& x_{1} \mathcal{T} x_{2} \Leftrightarrow\left(x_{1}, x_{2}\right) \in C_{>}^{2}, \\
& x_{1} \mathcal{F} x_{2} \Leftrightarrow\left(x_{1}, x_{2}\right) \in C^{2} \cap C^{1} .
\end{aligned}
$$


It is clear that asking for a representation in model (51) is equivalent to asking for the existence of two functions $f$ on $X_{1}$ and $g$ on $X_{2}$ such that

$$
\begin{aligned}
& x_{1} \mathcal{T} x_{2} \Leftrightarrow f\left(x_{1}\right)>g\left(x_{2}\right), \\
& x_{1} \mathcal{F} x_{2} \Leftrightarrow f\left(x_{1}\right)=g\left(x_{2}\right),
\end{aligned}
$$

and Proposition 12 gives necessary and sufficient conditions for that.

In view of the results in Levine (1970) (see also Krantz et al., 1971, sec. 6.7), it seems clear that the case of three categories and two attributes is also quite particular. This calls for the study of a generalization of the bi-semiorder model introduced in Ducamp and Falmagne (1969) in which two frontiers are introduced. This will be the subject of a subsequent paper.

A distinct motivation can be found in the study of time intervals in the context of temporal $\operatorname{logic}{ }^{5}$. In this field of study, an important question is to locate events ${ }^{6}$ on a time scale using only information linked to the fact one event entirely precedes another or that there is a period of time during which they simultaneously occur. Clearly, the results on the numerical representation of interval orders give a good basis to cope with such problems. In such a context, it may also be interesting to consider a relation saying that an event is immediately followed by another (see, e.g., Golumbic and Shamir, 1993). When this extra relation is brought into the picture, the question of locating events on a time scale can be solved using our results about the numerical representation of interval orders with frontier.

\section{Acknowledgments}

During the preparation of this paper, Denis Bouyssou was supported by the Belgian Fonds National de la Recherche Scientifique and Thierry Marchant benefited from a visiting position at the Université Paris Dauphine. This support is gratefully acknowledged. We wish to thank Meltem Öztürk for her detailed comments on an earlier draft of this text. We are also most grateful to the two anonymous referees and the action editor Jean-Paul Doignon for their extremely helpful comments and suggestions. The usual caveat applies.

\footnotetext{
${ }^{5}$ Recent references on this topic include Allen (1983, 1991), Allen and Hayes (1989) (in the area of Computer Science) and Lück (2006) (in the area of Philosophy). The problem of defining instants from "events" is old, dating back at least to Wiener (1914). Early developments of interval orders motivated by this problem are reviewed in Fishburn and Monjardet (1992).

${ }^{6}$ The term "event" is borrowed from the literature on temporal logic. These "events" should be thought as having a certain duration.
} 


\section{References}

Abrísqueta, F. J., Candeal, J. C., Induráin, E., and Zudaire, M. (2009). Scott-Suppes representability of semiorders: Internal conditions. Mathematical Social Sciences, $\mathbf{5 7}(2): 245-261$.

Aleskerov, F., Bouyssou, D., and Monjardet, B. (2007). Utility maximization, choice and preference. Springer-Verlag, Berlin, 2nd ed.

Allen, J. F. (1983). Maintaining knowledge about temporal intervals. Communications of the ACM, 26:832-843.

Allen, J. F. (1991). Time and time again: The many ways to represent time. International Journal of Intelligent Systems, 6:341-355.

Allen, J. F. and Hayes, P. (1989). Moments and points in an interval-based temporal logic. Computational Intelligence, 5:225-238.

Beja, A. and Gilboa, I. (1992). Numerical representations of imperfectly ordered preferences (a unified geometric exposition). Journal of Mathematical Psychology, 36:426449.

Bosi, B., Candeal, J. C., Induráin, E., Oloriz, E., and Zudaire, M. (2001). Numerical representations of interval orders. Order, 18:171-190.

Bouyssou, D. and Marchant, Th. (2007a). An axiomatic approach to noncompensatory sorting methods in MCDM, I: The case of two categories. European Journal of Operational Research, 178(1):217-245.

Bouyssou, D. and Marchant, Th. (2007b). An axiomatic approach to noncompensatory sorting methods in MCDM, II: More than two categories. European Journal of Operational Research, 178(1):246-276.

Bouyssou, D. and Marchant, Th. (2009). Ordered categories and additive conjoint measurement on connected sets. Journal of Mathematical Psychology, 53(2):92-105.

Bouyssou, D. and Marchant, Th. (2010). Additive conjoint measurement with ordered categories. European Journal of Operational Research, 203(1):195-204.

Bridges, D. S. (1983a). Numerical representation of intransitive preferences on a countable set. Journal of Economic Theory, 30:213-217.

Bridges, D. S. (1983b). A numerical representation of preferences with intransitive indifference. Journal of Mathematical Economics, 11:25-42.

Bridges, D. S. (1985). Representing interval orders by a single real-valued function. Journal of Economic Theory, 36:149-155.

Candeal, J. C., Induráin, E., and Zudaire, M. (2002). Numerical representability of semiorders. Mathematical Social Sciences, 43:61-77.

Doignon, J.-P., Ducamp, A., and Falmagne, J.-C. (1984). On realizable biorders and the biorder dimension of a relation. Journal of Mathematical Psychology, 28:73-109.

Doignon, J.-P., Ducamp, A., and Falmagne, J.-C. (1987). On the separation of two relations by a biorder or a semiorder. Mathematical Social Sciences, 13:1-18.

Doignon, J.-P. and Falmagne, J.-C. (1984). Matching relations and the dimensional structure of social choices. Mathematical Social Sciences, 7:211-229.

Doignon, J.-P., Monjardet, B., Roubens, M., and Vincke, Ph. (1988). Biorder fami- 
lies, valued relations and preference modelling. Journal of Mathematical Psychology, 30:435-480.

Ducamp, A. and Falmagne, J.-C. (1969). Composite measurement. Journal of Mathematical Psychology, 6:359-390.

Fishburn, P. C. (1970). Intransitive indifference with unequal indifference intervals. Journal of Mathematical Psychlogy, 7:144-149.

Fishburn, P. C. (1973). Interval representations for interval orders and semiorders. Journal of Mathematical Psychology, 10:91-105.

Fishburn, P. C. (1985). Interval orders and intervals graphs. Wiley, New York.

Fishburn, P. C. and Monjardet, B. (1992). Norbert Wiener on the theory of measurement (1914, 1915, 1921). Journal of Mathematical Psychology, 36:165-184.

Gensemer, S. H. (1987a). Continuous semiorder representations. Journal of Mathematical Economics, 16:275-289.

Gensemer, S. H. (1987b). On relationships between numerical representations of interval orders and semiorders. Journal of Economic Theory, 43:157-169.

Goldstein, W. M. (1991). Decomposable threshold models. Journal of Mathematical Psychology, 35:64-79.

Golumbic, M. C. and Shamir, R. (1993). Complexity and algorithms for reasoning about time: A graph-theoretic approach. Journal of the ACM, 40:1108-1133.

Krantz, D. H., Luce, R. D., Suppes, P., and Tversky, A. (1971). Foundations of measurement, vol. 1: Additive and polynomial representations. Academic Press, New York.

Levine, M. V. (1970). Transformations that render curves parallel. Journal of Mathematical Psychology, 7:410-443.

Luce, R. D. (1956). Semi-orders and a theory of utility discrimination. Econometrica, 24:178-191.

Lück, U. (2004). Representing interval orders by arbitrary real intervals. Tech. rep., Department of Philosophy, University of Munich.

Lück, U. (2006). Continu'ous time goes by Russell. Notre Dame Journal of Formal Logic, 47:397-434.

Manders, K. L. (1981). On JND representations of semiorders. Journal of Mathematical Psychology, 24:224-248.

Monjardet, B. (1978). Axiomatiques et propriétés des quasi-ordres. Mathématiques et Sciences Humaines, 63:51-82.

Nakamura, Y. (2002). Real interval representations. Journal of Mathematical Psychology, 46:140-177.

Narens, L. (1994). The measurement theory of dense threshold structures. Journal of Mathematical Psychology, 38:301-321.

Oloriz, E., Candeal, J. C., and Induráin, E. (1998). Representability of interval orders. Journal of Economic Theory, 78:219-227.

Pirlot, M. and Vincke, Ph. (1997). Semiorders. Properties, representations, applications. Kluwer, Dordrecht.

Roy, B. (1970). Algèbre moderne et théorie des graphes orientées vers les sciences 
économiques et sociales, vol. 2 : Applications et problèmes spécifiques. Dunod, Paris.

Roy, B. and Vincke, Ph. (1987). Pseudo-orders: Definition, properties and numerical representation. Mathematical Social Sciences, 14:263-274.

Schrijver, A. (2003). Combinatorial optimization: Polyhedra and efficiency. SpringerVerlag, Berlin.

Scott, D. and Suppes, P. (1958). Foundational aspects of theories of measurement. Journal of Symbolic Logic, 23:113-128.

Słowiński, R., Greco, S., and Matarazzo, B. (2002). Axiomatization of utility, outranking and decision-rule preference models for multiple-criteria classification problems under partial inconsistency with the dominance principle. Control and Cybernetics, 31(4):1005-1035.

Suppes, P., Krantz, D. H., Luce, R. D., and Tversky, A. (1989). Foundations of measurement, vol. 2: Geometrical, threshold, and probabilistic representations. Academic Press, New York.

Vincke, Ph. (1980). Vrai, quasi, pseudo et précritères dans un ensemble fini : propriétés et algorithmes. Cahier du LAMSADE 27, Université Paris-Dauphine, Paris.

Wiener, N. (1914). A contribution to the theory of relative position. Proceedings of the Cambridge Philosophical Society, 17:441-449. 